\title{
A Hybrid Demon Algorithm for the Two-Dimensional Orthogonal Strip Packing Problem
}

\author{
Bili Chen, ${ }^{1}$ Yong Wang, ${ }^{2}$ and Shuangyuan Yang1 \\ ${ }^{1}$ Software School, Xiamen University, Xiamen 361005, China \\ ${ }^{2}$ School of Economics and Business Administration, Chongqing Key Laboratory of Logistics, \\ Chongqing University, Chongqing 400044, China
}

Correspondence should be addressed to Yong Wang; wangyongkt@163.com

Received 3 September 2014; Accepted 23 December 2014

Academic Editor: Anders Eriksson

Copyright (C) 2015 Bili Chen et al. This is an open access article distributed under the Creative Commons Attribution License, which permits unrestricted use, distribution, and reproduction in any medium, provided the original work is properly cited.

\begin{abstract}
This paper develops a hybrid demon algorithm for a two-dimensional orthogonal strip packing problem. This algorithm combines a placement procedure based on an improved heuristic, local search, and demon algorithm involved in setting one parameter. The hybrid algorithm is tested on a wide set of benchmark instances taken from the literature and compared with other well-known algorithms. The computation results validate the quality of the solutions and the effectiveness of the proposed algorithm.
\end{abstract}

\section{Introduction}

Cutting and packing are a very active field of research within operational research, computer science, mathematics, and management science. The two-dimensional cutting and packing problem is widely applied in optimally cutting raw materials such as glass, textile, steel, and paper and transportation and logistics fields. For example, in textile or glass industries, rectangular components have to be cut from large sheets of material. In warehousing, goods have to be placed on shelves. In newspapers paging, articles and advertisements have to be arranged in pages [1]. In order to raise the profitability of the manufacturing or logistics company, the consumption of the raw materials or the cost of transportation should be minimized. Obviously, if an enterprise designs a production scheme using the least waste raw material, it can reduce the manufacturing costs and increase the product's competitiveness in the market.

The two-dimensional orthogonal strip packing problems (2SP) addressed in this paper consist of packing rectangular pieces into a large rectangular sheet of fixed width and unlimited height in order to minimize the used height, where the rectangular pieces are placed orthogonally without overlap and no rotations are allowed. This problem is of significance both from a theoretical and a practical point of view because it arises in various production processes and has many applications in the glass, steel, paper, and textile industries, and they also have indirect applications in other fields [2] such as layout designing, transportation, and logistics. More extensive survey and classification on cutting and packing problems may refer to Lodi et al. [1] and Wäscher et al. [3].

2SP is known to be NP-hard, some exact algorithms are proposed by Martello et al. [4] and Kenmochi et al. [5], but the size of instances that exact algorithms can handle tends to be small. Therefore, many heuristic algorithms have been suggested in the literature. Baker et al. [6] proposed a bottom-left-fill (BLF) algorithm for 2SP and variants of BLF [7]. Different types of construction heuristics have also been proposed recently, for example, the best-fit heuristic [8], a recursive heuristic [9], a bricklaying heuristic [10], a least waste heuristic [11], and a scoring heuristic [12].

In comparison to the literature on construction heuristic algorithms to packing problems, metaheuristic algorithms are paid more and more attention recently. Bortfeldt [13] proposed a genetic algorithm. The above two algorithms explore placements directly and allow infeasible solutions. However, most metaheuristic algorithms have been developed by incorporating a construction heuristic to improve the quality of solutions. Hopper and Turton [14] implemented 
TABLE 1: New scoring function score $\left(i, h_{1}, h_{2}, w\right)$ for $h_{1} \geq h_{2}$.

\begin{tabular}{|c|c|c|c|c|}
\hline & Conditions & $f$ & $f_{\text {new }}$ & $m$ \\
\hline \multirow{5}{*}{$h_{1} \geq h_{2}$} & Case (1): $w=r[i] \cdot$ width and $h_{1}=r[i] \cdot$ length & 4 & 4 & -2 or -1 \\
\hline & Case (2): $w=r[i] \cdot$ width and $h_{1}<r[i] \cdot$ length & 3 & 3 & 0 \\
\hline & Case (3): $w=r[i] \cdot$ width and $h_{1}>r[i] \cdot$ length & 2 & 1 & 0 \\
\hline & Case (4): $w>r[i] \cdot$ width and $h_{1}=r[i] \cdot$ length & 1 & 2 & 0 \\
\hline & Case (5): $w>r[i] \cdot$ width & 0 & 0 & +1 \\
\hline
\end{tabular}

a simulated annealing, tabu search, and genetic algorithm by incorporating BLF, respectively. Lesh et al. [15] proposed new heuristic and interactive approaches based on BLF for 2SP. Alvarez-Valdes et al. [16] presented a greedy randomized adaptive search procedure (GRASP) that involves learning some instances to determine the desirable parameter settings for 2SP. Wei et al. [11] presented a least waste algorithm by combining a simulated annealing algorithm for rectangle packing problem. Burke et al. [17] implemented a simulated annealing, tabu search, and genetic algorithm by incorporating BF. Leung et al. [18] proposed a simulated annealing algorithm based on a scoring rule heuristic. Some other algorithms based on different types of strategies have also been proposed, for example, SVC [19] and SWL [20]. Zhang et al. [21] developed a binary search heuristic algorithm based on randomized local search for the rectangular strip packing problem. In particular, an efficient algorithm for strip packing problem can be extended to solve other problems such as bin packing problem $[22,23]$. In this paper, we present a hybrid demon algorithm for 2SP which combines a demon algorithm with local search and an improved heuristic. This paper mainly has three contributions: firstly, a new scoring rule is presented; secondly, a least waste strategy is proposed; at last, a demon algorithm with fewer parameters than simulated annealing algorithm is applied to solve 2SP.

The remainder of the paper is organized as follows. Section 2 describes the hybrid algorithm: an improved scoring rule and demon algorithm. Section 3 investigates the effect of the parameter of demon algorithm and the least waste strategy. Section 4 reports the experimental results. Section 5 summarizes the conclusions and proposes future work.

\section{Hybrid Algorithm}

2.1. Improved Construction Heuristic Algorithm. It has been reported that construction heuristic algorithm is one of the best heuristics while combining with a simulated annealing algorithm [18]. Construction heuristic algorithm is stated as follows: give a rectangle piece sequence, the algorithm finds an unplaced piece with the maximum score for the lowest and the most left space, and then place it. Repeat the above process until all the pieces are placed. Leung et al. [18] proposed the scoring rules as Table 1 which is very important to select one unplaced piece, where $w$ is the width of the available space and $h_{1}$ and $h_{2}$ are the height of the left and right, respectively, wall of the available space. $r[i] \cdot$ width and $r[i] \cdot$ length denote width and length, respectively, of rectangular piece $i, m$ denotes the change number of spaces, and $f$ is fitness value as Leung et al. [18]. However, they do not explain why case (3) in Table 1 has higher score than case (4). In fact, the two cases have the same $m$; namely, the number of available spaces does not increase. Due to the fact that the objective of the problem is to minimize the height of the sheet, the result obtained by the scoring rule in Table 1 may be bad, while case (3) has higher score than case (4). For example, given a piece sequence: red, blue, yellow, and green, the packing result is shown in Figure 1, the yellow piece will be first placed according to the original scoring rule $f$ after the red piece and blue piece are placed, and then the green piece is placed as Figure 1(a), so the height obtained is 11. However, the height obtained is 9 if the new scoring rule $f_{\text {new }}$ is used that the green piece should be placed earlier than the yellow piece. The above example shows that the new scoring rule may lead to a better result than the original scoring rule. So the new scoring rule $f_{\text {new }}$ for $h_{1} \geq h_{2}$ as shown in Table 1 is used in this paper; for $h_{1}<h_{2}$, the new scoring rule $f_{\text {new }}$ can be calculated similarly.

In addition, one piece $r$ is selected by case (4) in Table 1, and we can try to place it into the current available space $s$; if it leads to the waste of the remainder space, then find another piece $q$ with the maximal width from all the unplaced pieces that meets $h_{q}=h_{r}$ and can be packed into $s$. For example, given a piece sequence: red, yellow, blue, green, black, and grey, the packing result is shown in Figure 2(a), the width of the current available space $s$ is 4 after the red, yellow, blue, and green pieces are placed, the black piece $r\left(h_{r}=5\right.$ and $w_{r}=2$ ) is selected by the scoring rule, and after it is placed, the width of the remainder space is 2 . If the width of all the unpacked piece is larger than 2 , the remainder space $2 \times 3$ will be wasted. The grey piece is placed after the black piece is placed. The height obtained is 13 . However, the obtained height is 11 if we use the least waste strategy. Namely, we can find another piece $q\left(h_{q}=5\right.$ and $\left.w_{q}=3\right)$ from all the unplaced pieces. Obviously, placing the piece $q$ makes waste least and obtains the smaller height. Similarly, if one piece $r$ is selected by case (5) in Table 1 , and placing it leads to the waste of the remainder space, then find another unplaced piece $q$ with the maximal width from all the unplaced pieces that meets $c_{q} \geq c_{r}$ and can be packed into $s$, where $c_{q}$ and $c_{r}$ denote the perimeter of the pieces $q$ and $r$, respectively.

2.2. Hybrid Demon Algorithm. The simulated annealing algorithm (SA) was invented to allow computer simulation of equilibria in statistical physics. It is a powerful randomized search algorithm, and the computational results have shown that ISA [18] based on SA is the best algorithm for 2SP. However, SA has to set the initial temperature, the annealing rate, and the Markov chain length. These parameters have 


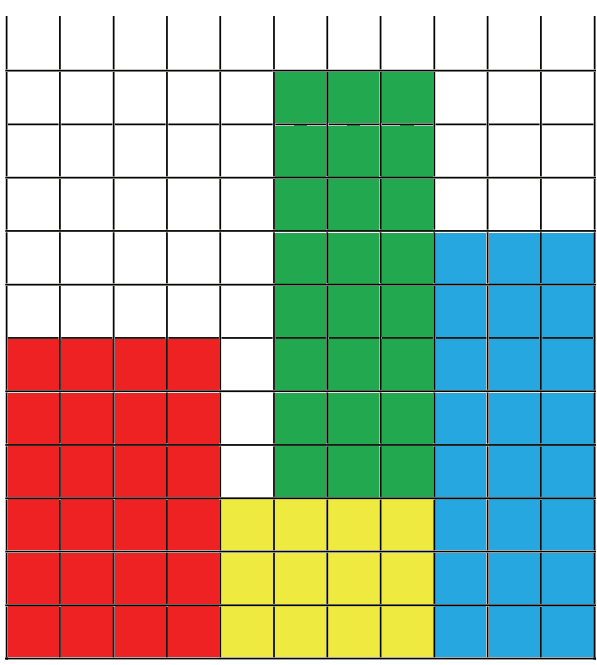

(a) Case (3) has higher score than case (4)

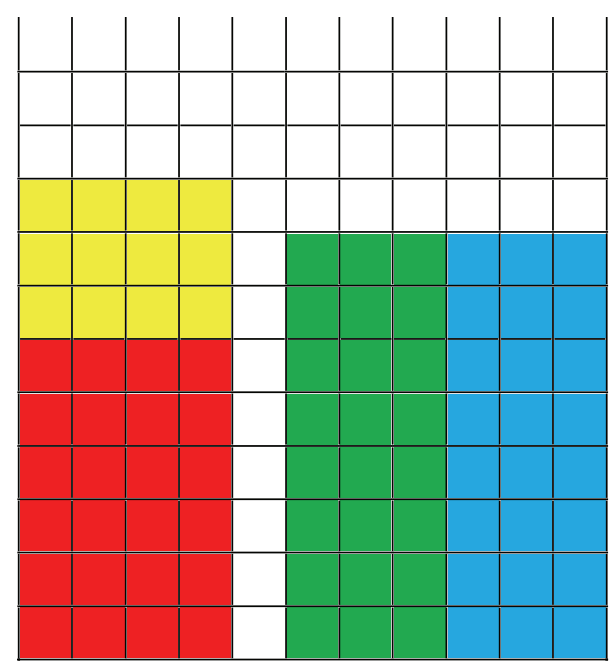

(b) Case (4) has higher score than case (3)

Figure 1: Placement by the different scoring rules.

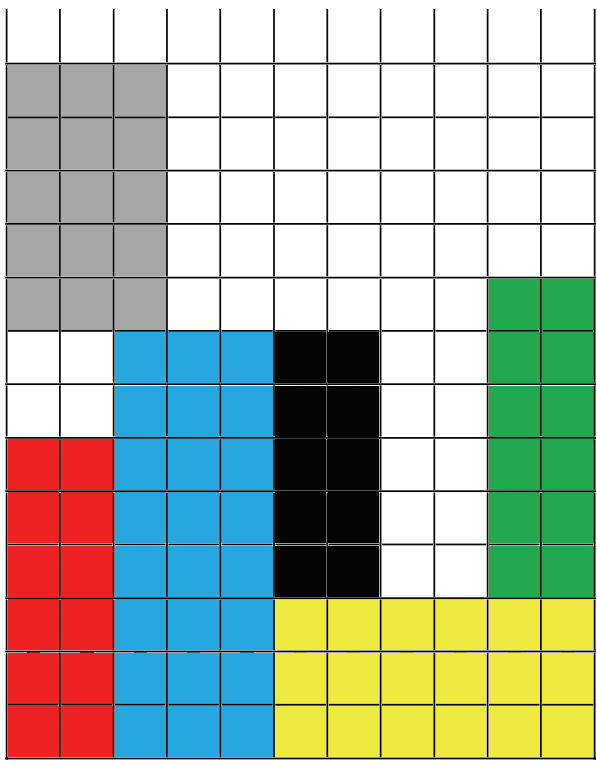

(a)

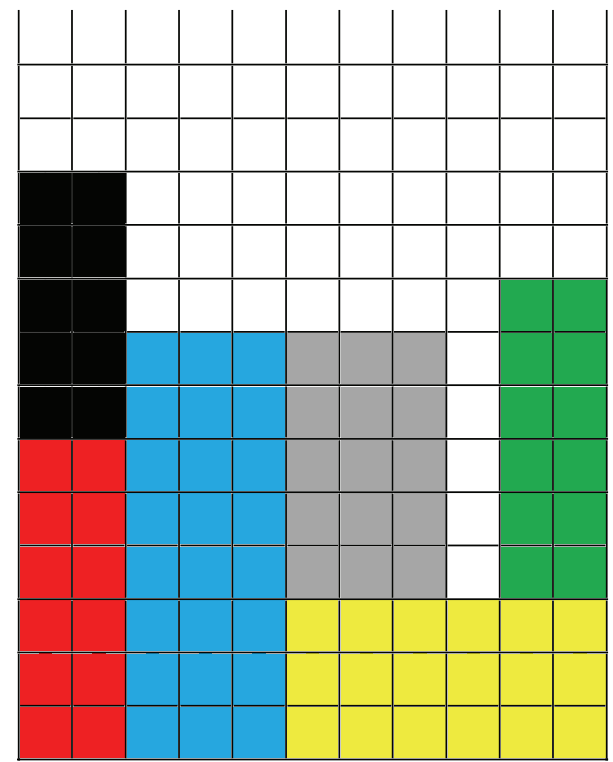

(b)

Figure 2: The least waste strategy.

significant effect on the performance of SA, but they have no general setting rule. Therefore, some variants with fewer parameters are paid attention by some researchers.

Demon algorithm is a simulated annealing based algorithm that uses computationally simpler acceptance function. This paper applies demon algorithm for 2SP. In order to solve it, the hybrid demon algorithm can be stated as in Algorithm 1, where LS() is similar to that in Leung et al. [18] and is stated as in Algorithm 2, where $L$ is the number of pieces, besth saves the best solution during the search process, and currenth is the height returned by HeuristicPacking $\left(X^{\prime}\right)$. The process of HeuristicPacking $\left(X^{\prime}\right)$ is the same as that of Leung et al. [18] except that a new scoring rule and a least waste strategy are used. Line 14 means keeping the old sequence $X$. $D$ is a key parameter that controls the acceptance function.

HDA first searches a better solution according to a local search algorithm LS() and then makes use of demon algorithm to improve the solution. Multistart ingredient (lines 1620 ) is employed to help HDA to escape from possible local minima.

\section{Effect of Parameter $D$}

HDA only involves in setting one parameter $D$, so it is simpler than simulated annealing algorithm. This section gives some 


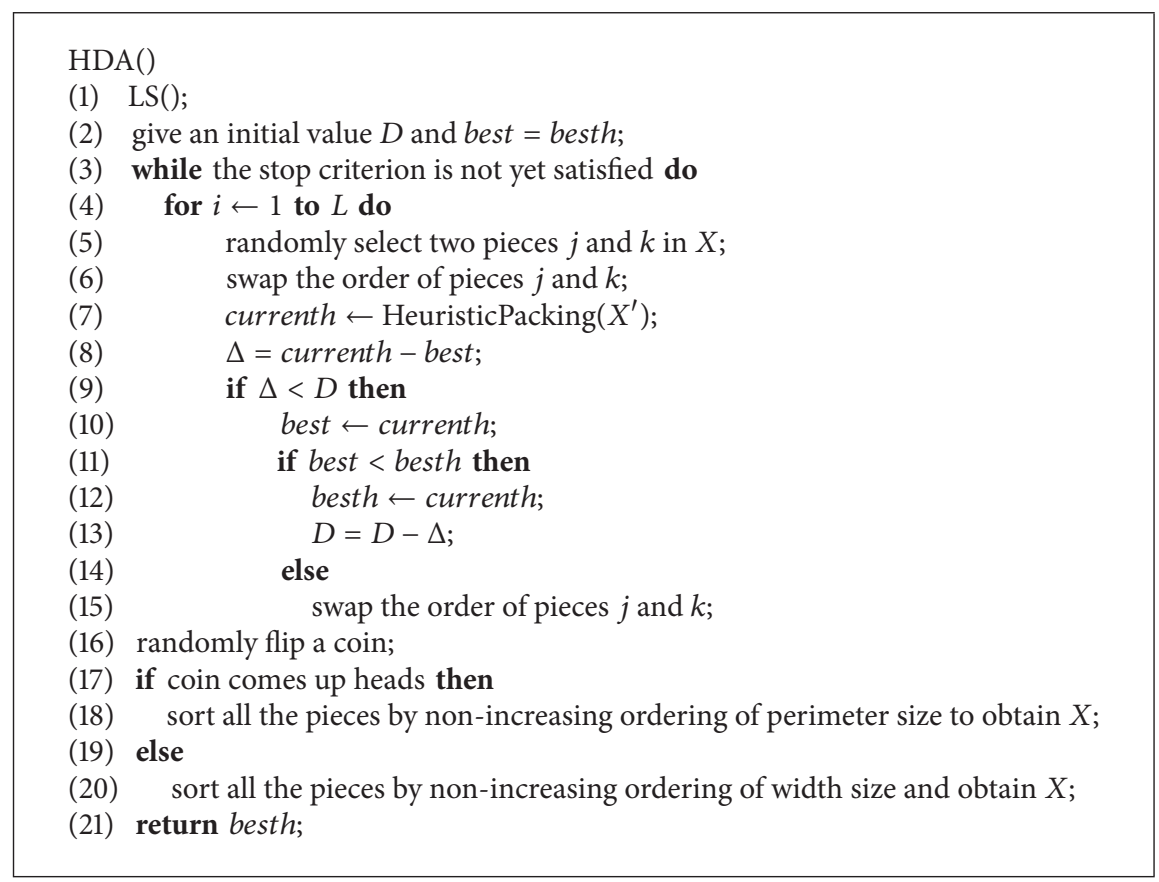

Algorithm 1: Hybrid demon algorithm.

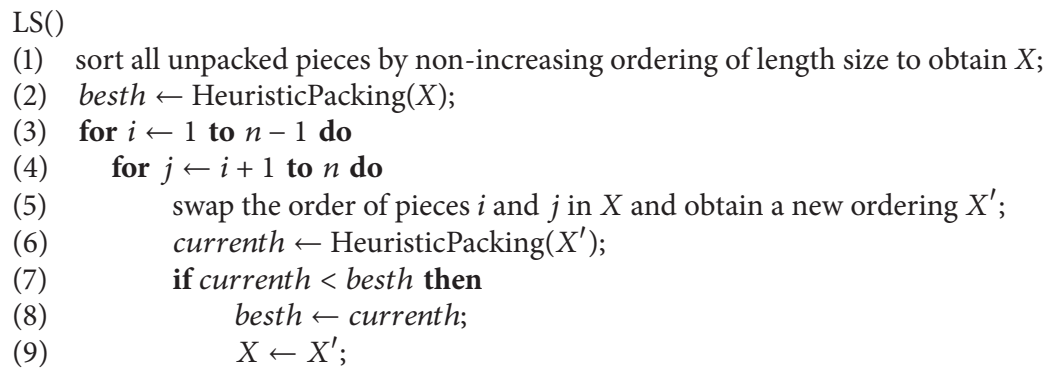

Algorithm 2

experiments to further select the value of $D$. In order to add the effect of demon algorithm, we only use some small and medium instances to save more time for demon algorithm to improve the solution. Several difficult data sets NT, gcut, Nicel 6, and Path1 6 are selected because they contain zerowaste instances and nonzero-waste instances. Figures 3 5 give the results of demon algorithm on the above instances, where $x$-axis denotes the different value of $D$ and $y$-axis denotes the average gap over 10 runs. Although there exists some slight difference for different data sets, we note that Gap will increase as $D$ increases. Therefore, $D=1$ is selected for the experiments in next section.

Figures 6, 7, and 8 report the results of hybrid demon algorithm with and without the least waste strategy, where $x$-axis denotes the problem instance and $y$-axis denotes the average gap over 10 runs. From these figures, we can note that the results obtained by the algorithm without the least waste strategy are slightly better than that obtained by the algorithm with the least waste strategy for zero-waste data

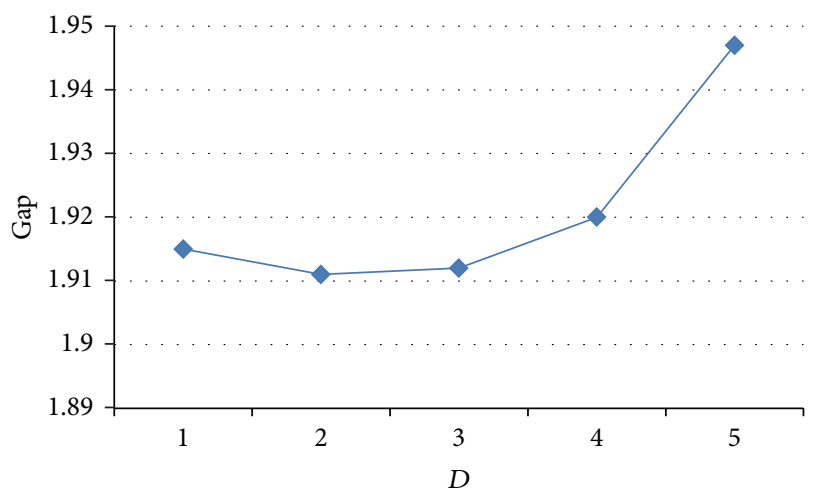

Figure 3: Effect of $D$ on the data set NT.

sets $\mathrm{C}$. The algorithm with the least waste strategy can obtain the better results than that without the least waste strategy for nonzero-waste data sets. Because nonzero-waste data sets 


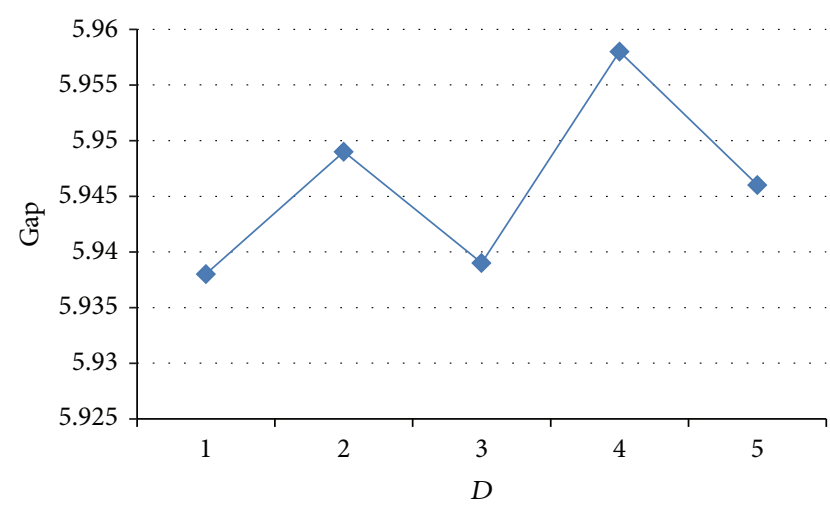

Figure 4: Effect of $D$ on the data set gcut.

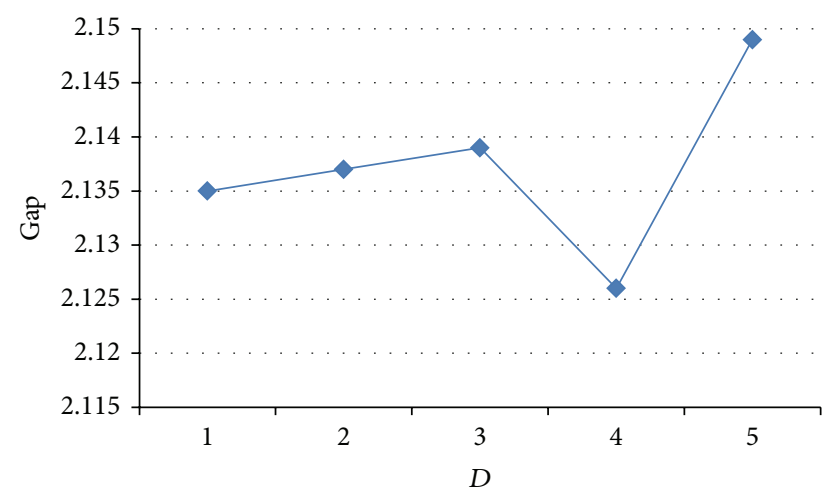

Figure 5: Effect of $D$ on the data set Nicel 6 and Path1 6.

contain more instances, so we use the least waste strategy in the experiments in next section.

\section{Experimental Results}

In this section, we present the results obtained in a set of experiments we conducted in order to evaluate the performance of the hybrid demon algorithm (HDA) proposed in this paper. This paper uses the same data sets $\mathrm{C}, \mathrm{N}, \mathrm{NT}$ and CX, 2sp, BWMV, Nice, and Path as Leung et al. [18]. All the data sets are publicly available at http://algorithm.xmu.edu .cn:10000/Download.aspx\#p4. These data sets include zerowaste instances and nonzero-waste instances from the literature. Zero-waste instances were created from known optimal solutions, and nonzero-waste instances do not necessarily have an optimal solution and their optimal solutions involve some waste regions.

The algorithm was implemented in Visual $\mathrm{C}++6.0$ and the experimental tests were run on a computer with an Intel core 2 CPU $2.13 \mathrm{GHz}$ and 0.99 GB RAM. GRASP [16], SVC [19], and ISA [18] are among the supposedly excellent algorithms in the current literature, so they are selected to compare with HDA. Computational results of GRASP, SVC, and ISA were taken from Leung et al. [18]. They were run on a computer with Intel Xeon CPU E5405 2.00 GHz 1.99 GB RAM and were run 10 times with a time limit of 60 seconds per run for each instance. HDA was allowed 60-second duration

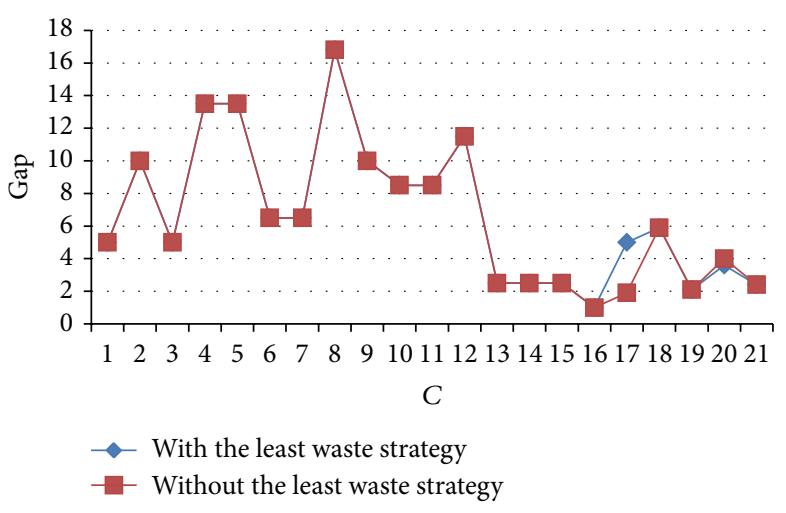

FIGURE 6: Effect of the least waste strategy on the data set $C$.

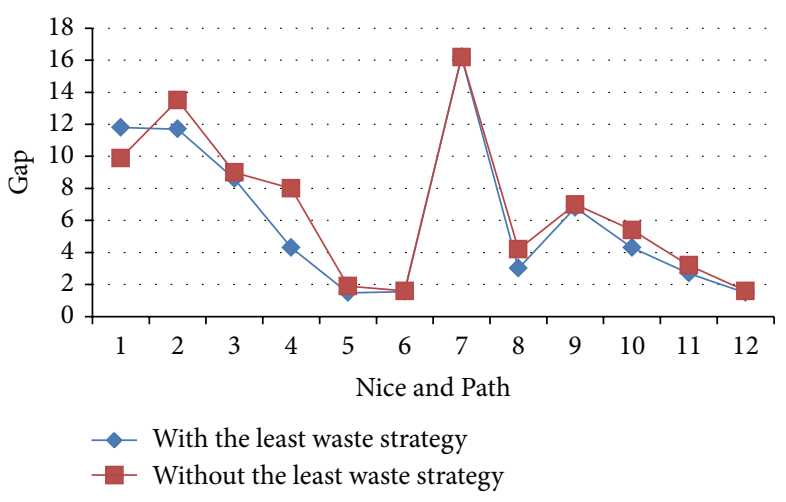

FIGURE 7: Effect of the least waste strategy on the data set Nicel 6 and Path1 6.

too. The computational results are reported in Tables 2 9, where Instance denotes problem instance, $n$ is the number of rectangular pieces, $W$ is the width of the rectangular sheet, LB is the optimal height for zero-waste instances and is the lower bound for nonzero-waste instances, and meanh denotes the average height obtained by each algorithm, running 10 times for each instance, respectively. Gap is defined as follows: Gap $=100 \times($ meanh $-\mathrm{LB}) / \mathrm{LB}$. The detailed results on the best and worst cases are available from the authors after this paper is published.

4.1. Zero-Waste Instances. Tables 2 9 report the results of four algorithms on zero-waste problem instances. In each table, the best results obtained by four algorithms are marked by boldface. The first four columns describe the characteristics of each instance. Columns 5 8 correspond to the average height obtained by GRASP, SVC, ISA, and HDA, respectively. Columns 9 12 correspond to the Gap obtained by GRASP, SVC, ISA, and HDA, respectively.

Table 2 shows the results of four algorithms for the data set $C$ [14], which have been used by many authors. From Table 2, GRASP, SVC, and ISA return an average gap of 0.95 , 1.03 , and 0.76 , respectively. HDA with an average gap of 0.71 performs better than GRASP, SVC, and ISA. In addition, we can observe that HDA performs well for large instances. 
TABLE 2: Results obtained by GRASP, SVC, ISA, and HDA on C.

\begin{tabular}{|c|c|c|c|c|c|c|c|c|c|c|c|}
\hline \multicolumn{4}{|c|}{ Instance } & \multicolumn{4}{|c|}{ meanh } & \multicolumn{4}{|c|}{ Gap } \\
\hline & $n$ & $W$ & LB & GRASP & SVC & ISA & HDA & GRASP & SVC & ISA & HDA \\
\hline $\mathrm{C} 11$ & 16 & 20 & 20 & 20 & 20 & 20.0 & 20 & 0.0 & 0.0 & 0.0 & 0.0 \\
\hline $\mathrm{C} 12$ & 17 & 20 & 20 & 20 & 21 & 20.0 & 20 & 0.0 & 5.0 & 0.0 & 0.0 \\
\hline $\mathrm{C} 13$ & 16 & 20 & 20 & 20 & 20 & 20.0 & 20 & 0.0 & 0.0 & 0.0 & 0.0 \\
\hline $\mathrm{C} 21$ & 25 & 40 & 15 & 15 & 15 & 15.0 & 15 & 0.0 & 0.0 & 0.0 & 0.0 \\
\hline $\mathrm{C} 22$ & 25 & 40 & 15 & 15 & 15 & 15.0 & 15 & 0.0 & 0.0 & 0.0 & 0.0 \\
\hline $\mathrm{C} 23$ & 25 & 40 & 15 & 15 & 15 & 15.0 & 15 & 0.0 & 0.0 & 0.0 & 0.0 \\
\hline C31 & 28 & 60 & 30 & 30 & 30 & 30.0 & 30 & 0.0 & 0.0 & 0.0 & 0.0 \\
\hline $\mathrm{C} 32$ & 29 & 60 & 30 & 31 & 31 & 31.0 & 30.9 & 3.3 & 3.3 & 3.3 & 3.0 \\
\hline $\mathrm{C} 33$ & 28 & 60 & 30 & 30 & 30 & 30.0 & 30 & 0.0 & 0.0 & 0.0 & 0.0 \\
\hline C41 & 49 & 60 & 60 & 61 & 61 & 61.0 & 61 & 1.7 & 1.7 & 1.7 & 1.7 \\
\hline C42 & 49 & 60 & 60 & 61 & 61 & 61.0 & 61 & 1.7 & 1.7 & 1.7 & 1.7 \\
\hline $\mathrm{C} 43$ & 49 & 60 & 60 & 61 & 61 & 60.9 & 61 & 1.7 & 1.7 & 1.5 & 1.7 \\
\hline C51 & 73 & 60 & 90 & 91 & 91 & 91.0 & 91 & 1.1 & 1.1 & 1.1 & 1.1 \\
\hline C52 & 73 & 60 & 90 & 91 & 91 & 90.8 & 91 & 1.1 & 1.1 & 0.9 & 1.1 \\
\hline C53 & 73 & 60 & 90 & 91 & 91 & 91.0 & 91 & 1.1 & 1.1 & 1.1 & 1.1 \\
\hline C61 & 97 & 80 & 120 & 122 & 121 & 121.0 & 121 & 1.7 & 0.8 & 0.8 & 0.8 \\
\hline C62 & 97 & 80 & 120 & 121 & 121 & 121.0 & 121 & 0.8 & 0.8 & 0.8 & 0.8 \\
\hline C63 & 97 & 80 & 120 & 122 & 121 & 121.0 & 121 & 1.7 & 0.8 & 0.8 & 0.8 \\
\hline C71 & 196 & 160 & 240 & 244 & 242 & 242.0 & 241 & 1.7 & 0.8 & 0.8 & 0.4 \\
\hline C72 & 197 & 160 & 240 & 243 & 242 & 241.0 & 241 & 1.3 & 0.8 & 0.4 & 0.4 \\
\hline $\mathrm{C} 73$ & 196 & 160 & 240 & 243 & 242 & 242.0 & 241 & 1.3 & 0.8 & 0.8 & 0.4 \\
\hline \multicolumn{4}{|c|}{ Average } & 83.19 & 82.95 & 82.84 & 82.76 & 0.95 & 1.03 & 0.76 & 0.71 \\
\hline
\end{tabular}

TABLE 3: Results obtained by GRASP, SVC, ISA, and HDA on N.

\begin{tabular}{|c|c|c|c|c|c|c|c|c|c|c|c|}
\hline \multicolumn{4}{|c|}{ Instance } & \multicolumn{4}{|c|}{ meanh } & \multicolumn{4}{|c|}{ Gap } \\
\hline & $n$ & $W$ & LB & GRASP & SVC & ISA & HDA & GRASP & SVC & ISA & HDA \\
\hline N1 & 10 & 40 & 40 & 40 & 40 & 40.0 & 40 & 0.0 & 0.0 & 0.0 & 0.0 \\
\hline N2 & 20 & 30 & 50 & 50 & 50 & 50.0 & 50 & 0.0 & 0.0 & 0.0 & 0.0 \\
\hline N3 & 30 & 30 & 50 & 51 & 50 & 50.1 & 50 & 2.0 & 0.0 & 0.2 & 0.0 \\
\hline N4 & 40 & 80 & 80 & 81 & 81 & 80.0 & 80 & 1.3 & 1.3 & 0.0 & 0.0 \\
\hline N5 & 50 & 100 & 100 & 102 & 101 & 101.0 & 100 & 2.0 & 1.0 & 1.0 & 0.0 \\
\hline N6 & 60 & 50 & 100 & 101 & 101 & 100.9 & 100.7 & 1.0 & 1.0 & 0.9 & 0.7 \\
\hline N7 & 70 & 80 & 100 & 101 & 101 & 100.0 & 100 & 1.0 & 1.0 & 0.0 & 0.0 \\
\hline N8 & 80 & 100 & 80 & 81 & 81 & 81.0 & 81 & 1.3 & 1.3 & 1.3 & 1.3 \\
\hline N9 & 100 & 50 & 150 & 151 & 151 & 150.9 & 151 & 0.7 & 0.7 & 0.6 & 0.7 \\
\hline N10 & 200 & 70 & 150 & 151 & 151 & 150.8 & 151 & 0.7 & 0.7 & 0.5 & 0.7 \\
\hline N11 & 300 & 70 & 150 & 151 & 151 & 150.7 & 150.8 & 0.7 & 0.7 & 0.5 & 0.5 \\
\hline N12 & 500 & 100 & 300 & 304 & 301 & 301.0 & 301 & 1.3 & 0.3 & 0.3 & 0.3 \\
\hline N13 & 3152 & 640 & 960 & 965 & 963 & 960.0 & 960 & 0.5 & 0.3 & 0.0 & 0.0 \\
\hline \multicolumn{4}{|c|}{ Average } & 179.15 & 178.62 & 178.18 & 178.12 & 0.95 & 0.63 & 0.41 & 0.32 \\
\hline
\end{tabular}

Table 3 shows the results of four algorithms for the data set $\mathrm{N}$ generated by Burke et al. [8]. From Table 3, GRASP, SVC, and ISA return an average gap of $0.95,0.63$, and 0.41 , respectively. HDA with an average gap of 0.32 performs better than GRASP, SVC, and ISA. What is more, the optimal solution of N13 is obtained by ISA and HDA.

Table 4 shows the results of four algorithms for the data set NT, which is generated by Hopper and Turton [14]. From
Table 4, GRASP, SVC, and ISA return an average gap of 2.32, 2.27, and 2.24, respectively. HDA with an average gap of 1.91 performs better than GRASP, SVC, and ISA. In addition, we can observe that HDA performs the same or better for large instances $\mathrm{n} 7 \mathrm{a} \sim \mathrm{e}$ and $\mathrm{t} 7 \mathrm{a} \sim \mathrm{e}$.

Table 5 shows the results of four algorithms for the extralarge data set CX, which is generated by Pinto and Oliveira [24]. From Table 5, GRASP, SVC, and ISA return an average 
TABLE 4: Results obtained by GRASP, SVC, ISA, and HDA on NT.

\begin{tabular}{|c|c|c|c|c|c|c|c|c|c|c|c|}
\hline \multicolumn{4}{|c|}{ Instance } & \multicolumn{4}{|c|}{ meanh } & \multicolumn{4}{|c|}{ Gap } \\
\hline & $n$ & $W$ & LB & GRASP & SVC & ISA & HDA & GRASP & SVC & ISA & HDA \\
\hline nla & 17 & 200 & 200 & 200 & 202 & 200.0 & 200 & 0.0 & 1.0 & 0.0 & 0.0 \\
\hline $\mathrm{nlb}$ & 17 & 200 & 200 & 209 & 200 & 211.2 & 200 & 4.5 & 0.0 & 5.6 & 0.0 \\
\hline nlc & 17 & 200 & 200 & 200 & 200 & 200.0 & 200 & 0.0 & 0.0 & 0.0 & 0.0 \\
\hline nld & 17 & 200 & 200 & 200 & 200 & 200.0 & 200 & 0.0 & 0.0 & 0.0 & 0.0 \\
\hline nle & 17 & 200 & 200 & 200 & 200 & 200.0 & 200 & 0.0 & 0.0 & 0.0 & 0.0 \\
\hline $\mathrm{n} 2 \mathrm{a}$ & 25 & 200 & 200 & 206 & 205 & 204.0 & 201.3 & 3.0 & 2.5 & 2.0 & 0.7 \\
\hline $\mathrm{n} 2 \mathrm{~b}$ & 25 & 200 & 200 & 206 & 209 & 209.4 & 210 & 3.0 & 4.5 & 4.7 & 5.0 \\
\hline $\mathrm{n} 2 \mathrm{c}$ & 25 & 200 & 200 & 208 & 209 & 208.5 & 206.3 & 4.0 & 4.5 & 4.3 & 3.2 \\
\hline $\mathrm{n} 2 \mathrm{~d}$ & 25 & 200 & 200 & 209 & 207 & 207.8 & 205.9 & 4.5 & 3.5 & 3.9 & 3.0 \\
\hline $\mathrm{n} 2 \mathrm{e}$ & 25 & 200 & 200 & 206 & 205 & 206.7 & 206.1 & 3.0 & 2.5 & 3.3 & 3.1 \\
\hline n3a & 29 & 200 & 200 & 209 & 208 & 206.1 & 206.3 & 4.5 & 4.0 & 3.1 & 3.2 \\
\hline $\mathrm{n} 3 \mathrm{~b}$ & 29 & 200 & 200 & 208 & 207 & 209.0 & 208.9 & 4.0 & 3.5 & 4.5 & 4.5 \\
\hline $\mathrm{n} 3 \mathrm{c}$ & 29 & 200 & 200 & 205 & 207 & 206.1 & 205 & 2.5 & 3.5 & 3.1 & 2.5 \\
\hline $\mathrm{n} 3 \mathrm{~d}$ & 29 & 200 & 200 & 207 & 208 & 204.3 & 204.5 & 3.5 & 4.0 & 2.2 & 2.3 \\
\hline $\mathrm{n} 3 \mathrm{e}$ & 29 & 200 & 200 & 207 & 207 & 208.0 & 208.1 & 3.5 & 3.5 & 4.0 & 4.1 \\
\hline $\mathrm{n} 4 \mathrm{a}$ & 49 & 200 & 200 & 206 & 205 & 206.0 & 205.9 & 3.0 & 2.5 & 3.0 & 3.0 \\
\hline $\mathrm{n} 4 \mathrm{~b}$ & 49 & 200 & 200 & 207 & 205 & 205.0 & 204.7 & 3.5 & 2.5 & 2.5 & 2.3 \\
\hline $\mathrm{n} 4 \mathrm{c}$ & 49 & 200 & 200 & 205 & 205 & 206.0 & 205.3 & 2.5 & 2.5 & 3.0 & 2.7 \\
\hline $\mathrm{n} 4 \mathrm{~d}$ & 49 & 200 & 200 & 206 & 205 & 204.8 & 204.9 & 3.0 & 2.5 & 2.4 & 2.5 \\
\hline $\mathrm{n} 4 \mathrm{e}$ & 49 & 200 & 200 & 205 & 205 & 206.0 & 206.1 & 2.5 & 2.5 & 3.0 & 3.1 \\
\hline n5a & 73 & 200 & 200 & 205 & 204 & 205.1 & 205.5 & 2.5 & 2.0 & 2.6 & 2.8 \\
\hline $\mathrm{n} 5 \mathrm{~b}$ & 73 & 200 & 200 & 204 & 204 & 203.6 & 203.1 & 2.0 & 2.0 & 1.8 & 1.6 \\
\hline $\mathrm{n} 5 \mathrm{c}$ & 73 & 200 & 200 & 206 & 204 & 204.4 & 204.5 & 3.0 & 2.0 & 2.2 & 2.3 \\
\hline $\mathrm{n} 5 \mathrm{~d}$ & 73 & 200 & 200 & 204 & 205 & 205.0 & 204.7 & 2.0 & 2.5 & 2.5 & 2.3 \\
\hline $\mathrm{n} 5 \mathrm{e}$ & 73 & 200 & 200 & 206 & 205 & 204.7 & 204.9 & 3.0 & 2.5 & 2.3 & 2.5 \\
\hline n6a & 97 & 200 & 200 & 204 & 203 & 202.8 & 202.7 & 2.0 & 1.5 & 1.4 & 1.3 \\
\hline $\mathrm{n} 6 \mathrm{~b}$ & 97 & 200 & 200 & 204 & 204 & 203.0 & 202.9 & 2.0 & 2.0 & 1.5 & 1.5 \\
\hline $\mathrm{n} 6 \mathrm{c}$ & 97 & 200 & 200 & 204 & 204 & 203.6 & 203.2 & 2.0 & 2.0 & 1.8 & 1.6 \\
\hline $\mathrm{n} 6 \mathrm{~d}$ & 97 & 200 & 200 & 204.1 & 202 & 203.8 & 203 & 2.1 & 1.0 & 1.9 & 1.5 \\
\hline n6e & 97 & 200 & 200 & 204 & 203 & 203.5 & 203 & 2.0 & 1.5 & 1.8 & 1.5 \\
\hline $\mathrm{n} 7 \mathrm{a}$ & 199 & 200 & 200 & 202 & 202 & 201.0 & 201 & 1.0 & 1.0 & 0.5 & 0.5 \\
\hline $\mathrm{n} 7 \mathrm{~b}$ & 199 & 200 & 200 & 203 & 202 & 202.0 & 201 & 1.5 & 1.0 & 1.0 & 0.5 \\
\hline $\mathrm{n} 7 \mathrm{c}$ & 199 & 200 & 200 & 203 & 202 & 201.9 & 201 & 1.5 & 1.0 & 1.0 & 0.5 \\
\hline $\mathrm{n} 7 \mathrm{~d}$ & 199 & 200 & 200 & 203 & 202 & 201.9 & 201 & 1.5 & 1.0 & 1.0 & 0.5 \\
\hline $\mathrm{n} 7 \mathrm{e}$ & 199 & 200 & 200 & 203 & 202 & 201.9 & 201 & 1.5 & 1.0 & 1.0 & 0.5 \\
\hline tla & 17 & 200 & 200 & 200 & 200 & 200.0 & 200 & 0.0 & 0.0 & 0.0 & 0.0 \\
\hline $\mathrm{t} 1 \mathrm{~b}$ & 17 & 200 & 200 & 200 & 211 & 200.0 & 200 & 0.0 & 5.5 & 0.0 & 0.0 \\
\hline tlc & 17 & 200 & 200 & 200 & 210 & 200.0 & 200 & 0.0 & 5.0 & 0.0 & 0.0 \\
\hline tld & 17 & 200 & 200 & 200 & 200 & 211.8 & 200 & 0.0 & 0.0 & 5.9 & 0.0 \\
\hline tle & 17 & 200 & 200 & 200 & 209 & 200.0 & 200 & 0.0 & 4.5 & 0.0 & 0.0 \\
\hline $\mathrm{t} 2 \mathrm{a}$ & 25 & 200 & 200 & 204 & 207 & 207.0 & 206.3 & 2.0 & 3.5 & 3.5 & 3.2 \\
\hline $\mathrm{t} 2 \mathrm{~b}$ & 25 & 200 & 200 & 208 & 205 & 207.0 & 205.8 & 4.0 & 2.5 & 3.5 & 2.9 \\
\hline $\mathrm{t} 2 \mathrm{c}$ & 25 & 200 & 200 & 208 & 206 & 206.0 & 207.4 & 4.0 & 3.0 & 3.0 & 3.7 \\
\hline $\mathrm{t} 2 \mathrm{~d}$ & 25 & 200 & 200 & 206 & 207 & 209.3 & 204.4 & 3.0 & 3.5 & 4.7 & 2.2 \\
\hline $\mathrm{t} 2 \mathrm{e}$ & 25 & 200 & 200 & 206 & 207 & 207.4 & 205.9 & 3.0 & 3.5 & 3.7 & 3.0 \\
\hline $\mathrm{t} 3 \mathrm{a}$ & 29 & 200 & 200 & 207 & 208 & 209.0 & 209 & 3.5 & 4.0 & 4.5 & 4.5 \\
\hline$t 3 b$ & 29 & 200 & 200 & 209 & 207 & 208.1 & 207.9 & 4.5 & 3.5 & 4.1 & 4.0 \\
\hline $\mathrm{t} 3 \mathrm{c}$ & 29 & 200 & 200 & 206 & 207 & 206.6 & 206.3 & 3.0 & 3.5 & 3.3 & 3.2 \\
\hline $\mathrm{t} 3 \mathrm{~d}$ & 29 & 200 & 200 & 207 & 208 & 206.4 & 206.4 & 3.5 & 4.0 & 3.2 & 3.2 \\
\hline $\mathrm{t} 3 \mathrm{e}$ & 29 & 200 & 200 & 208 & 206 & 205.0 & 205 & 4.0 & 3.0 & 2.5 & 2.5 \\
\hline
\end{tabular}


TABLE 4: Continued.

\begin{tabular}{|c|c|c|c|c|c|c|c|c|c|c|c|}
\hline \multicolumn{4}{|c|}{ Instance } & \multicolumn{4}{|c|}{ meanh } & \multicolumn{4}{|c|}{ Gap } \\
\hline & $n$ & $W$ & LB & GRASP & SVC & ISA & HDA & GRASP & SVC & ISA & HDA \\
\hline $\mathrm{t} 4 \mathrm{a}$ & 49 & 200 & 200 & 205 & 205 & 205.0 & 204.7 & 2.5 & 2.5 & 2.5 & 2.3 \\
\hline $\mathrm{t} 4 \mathrm{~b}$ & 49 & 200 & 200 & 205 & 205 & 206.1 & 205.9 & 2.5 & 2.5 & 3.1 & 3.0 \\
\hline $\mathrm{t} 4 \mathrm{c}$ & 49 & 200 & 200 & 206 & 205 & 204.9 & 204.7 & 3.0 & 2.5 & 2.5 & 2.3 \\
\hline $\mathrm{t} 4 \mathrm{~d}$ & 49 & 200 & 200 & 206 & 205 & 205.7 & 205.4 & 3.0 & 2.5 & 2.8 & 2.7 \\
\hline $\mathrm{t} 4 \mathrm{e}$ & 49 & 200 & 200 & 207 & 205 & 205.2 & 205.5 & 3.5 & 2.5 & 2.6 & 2.8 \\
\hline $\mathrm{t} 5 \mathrm{a}$ & 73 & 200 & 200 & 206 & 204 & 204.4 & 204.5 & 3.0 & 2.0 & 2.2 & 2.3 \\
\hline $\mathrm{t} 5 \mathrm{~b}$ & 73 & 200 & 200 & 204 & 204 & 204.0 & 204.4 & 2.0 & 2.0 & 2.0 & 2.2 \\
\hline $\mathrm{t} 5 \mathrm{c}$ & 73 & 200 & 200 & 205 & 204 & 205.0 & 205.5 & 2.5 & 2.0 & 2.5 & 2.8 \\
\hline $\mathrm{t} 5 \mathrm{~d}$ & 73 & 200 & 200 & 204 & 205 & 204.9 & 204.7 & 2.0 & 2.5 & 2.5 & 2.3 \\
\hline $\mathrm{t} 5 \mathrm{e}$ & 73 & 200 & 200 & 204 & 204 & 204.0 & 204.7 & 2.0 & 2.0 & 2.0 & 2.3 \\
\hline t6a & 97 & 200 & 200 & 204 & 204 & 203.2 & 203.7 & 2.0 & 2.0 & 1.6 & 1.8 \\
\hline $\mathrm{t} 6 \mathrm{~b}$ & 97 & 200 & 200 & 204 & 202 & 203.4 & 203.2 & 2.0 & 1.0 & 1.7 & 1.6 \\
\hline t6c & 97 & 200 & 200 & 204 & 204 & 203.0 & 202.7 & 2.0 & 2.0 & 1.5 & 1.3 \\
\hline t6d & 97 & 200 & 200 & 204 & 204 & 203.5 & 203.7 & 2.0 & 2.0 & 1.8 & 1.8 \\
\hline t6e & 97 & 200 & 200 & 205 & 204 & 203.5 & 203.5 & 2.5 & 2.0 & 1.8 & 1.8 \\
\hline $\mathrm{t} 7 \mathrm{a}$ & 199 & 200 & 200 & 203 & 201 & 201.2 & 201 & 1.5 & 0.5 & 0.6 & 0.5 \\
\hline $\mathrm{t} 7 \mathrm{~b}$ & 199 & 200 & 200 & 203 & 202 & 201.0 & 201 & 1.5 & 1.0 & 0.5 & 0.5 \\
\hline $\mathrm{t} 7 \mathrm{c}$ & 199 & 200 & 200 & 204 & 202 & 201.0 & 201 & 2.0 & 1.0 & 0.5 & 0.5 \\
\hline $\mathrm{t} 7 \mathrm{~d}$ & 199 & 200 & 200 & 202 & 202 & 202.0 & 201 & 1.0 & 1.0 & 1.0 & 0.5 \\
\hline t7e & 199 & 200 & 200 & 203 & 202 & 201.7 & 201 & 1.5 & 1.0 & 0.8 & 0.5 \\
\hline & & & & 204.64 & 204.54 & 204.48 & 203.83 & 2.32 & 2.27 & 2.24 & 1.91 \\
\hline
\end{tabular}

TABLE 5: Results obtained by GRASP, SVC, ISA, and HDA on CX.

\begin{tabular}{lccccccccccc}
\hline & \multicolumn{1}{c}{ Instance } & \multicolumn{4}{c}{ meanh } & \multicolumn{3}{c}{ Gap } \\
& $n$ & $W$ & LB & GRASP & SVC & ISA & HDA & GRASP & SVC & ISA & HDA \\
\hline $50 \mathrm{cx}$ & 50 & 400 & 600 & 613 & 603 & 620.2 & 607.3 & 2.2 & 0.5 & 3.4 \\
$100 \mathrm{cx}$ & 100 & 400 & 600 & 617 & 616 & 615.8 & 617.3 & 2.8 & 2.7 & 2.6 & 2.9 \\
$500 \mathrm{cx}$ & 500 & 400 & 600 & 605 & 604 & 601.0 & 601 & 0.8 & 0.7 & 0.2 & 0.2 \\
$1000 \mathrm{cx}$ & 1000 & 400 & 600 & 602 & 601 & 600.0 & 600 & 0.3 & 0.2 & 0.0 & 0.0 \\
$5000 \mathrm{cx}$ & 5000 & 400 & 600 & 600 & 600 & 600.0 & 600 & 0.0 & 0.0 & 0.0 & 0.0 \\
$10000 \mathrm{cx}$ & 10000 & 400 & 600 & 600 & 600 & 600.0 & 600 & 0.0 & 0.0 & 0.0 & 0.0 \\
$15000 \mathrm{cx}$ & 15000 & 400 & 600 & 600 & 600 & 600.0 & 600 & 0.0 & 0.0 & 0.0 & 0.0 \\
\hline \multicolumn{1}{c}{} & Average & & 605.29 & $\mathbf{6 0 3 . 4 3}$ & 605.29 & 603.66 & 0.88 & $\mathbf{0 . 5 7}$ & 0.88 & 0.61 \\
\hline
\end{tabular}

gap of $0.88,0.57$, and 0.88 , respectively. HDA with an average gap of 0.61 performs better than GRASP and ISA and slightly worse than SVC.

4.2. Nonzero-Waste Instances. Section 4.1 has discussed the results on the zero-waste instances with known optimal solutions. In some practical applications, the optimal solutions often include some wasted regions. So nonzero-waste instances are more general and are widely used in the literature. We can compute the lower bound of these instances, and the optimal solutions of some instances are known because they are confirmed by other algorithms. In this section, we investigate if HDA can still be successfully employed to solve such instances. Tables 6 9 summarize the computational results on nonzero-waste instances.

Table 6 shows the results of nonzero-waste data sets $2 \mathrm{sp}$ which include ngcut [25], gcut [26], cgcut [27], and Beng [28]. The problem size of $2 \mathrm{sp}$ is very small; namely, $n$ is less than
200, so it is used by many authors to test the performance of the algorithms. From Table 6, GRASP, SVC, and ISA return an average gap of 2.68, 2.8, and 3.02, respectively. HDA with an average gap of 3.04 performs slightly worse than GRASP, SVC, and ISA. However, HDA can obtain smaller average meanh 1538.16 than GRASP, SVC, and ISA, so HDA is still efficient for $2 \mathrm{sp}$.

Table 7 shows the results of nonzero-waste data sets BWMV which include C01 C06 [29], C07 C10 [30]. The problem size of BWMV is very small $(n \leq 100)$, so it is used by many authors to test the performance of the algorithms. From Table 7, GRASP, SVC, and ISA return an average gap of $1.77,1.80$, and 1.66 , respectively. HDA with an average gap of 1.63 performs better than GRASP, SVC, and ISA. So HDA is superior to GRASP, SVC, and ISA.

Table 8 shows the results of data sets Nice and Path which are floating-point data sets and are generated by Valenzuela and Wang [31], where Nicel Nice5t are the sets of similarly 
TABLE 6: Results obtained by GRASP, SVC, ISA, and HDA on 2sp.

\begin{tabular}{|c|c|c|c|c|c|c|c|c|c|c|c|}
\hline \multicolumn{4}{|c|}{ Instance } & \multicolumn{4}{|c|}{ meanh } & \multicolumn{4}{|c|}{ Gap } \\
\hline & $n$ & $W$ & LB & GRASP & SVC & ISA & HDA & GRASP & SVC & ISA & HDA \\
\hline cgcutl & 16 & 10 & 23 & 23 & 23 & 23.0 & 24 & 0.0 & 0.0 & 0.0 & 4.3 \\
\hline cgcut 2 & 23 & 70 & 63 & 65 & 65 & 65.0 & 65 & 3.2 & 3.2 & 3.2 & 3.2 \\
\hline cgcut3 & 62 & 70 & 636 & 661 & 661 & 660.2 & 662.2 & 3.9 & 3.9 & 3.8 & 4.1 \\
\hline gcutl & 10 & 250 & 1016 & 1016 & 1016 & 1016.0 & 1016 & 0.0 & 0.0 & 0.0 & 0.0 \\
\hline gcut2 & 20 & 250 & 1133 & 1191 & 1187 & 1187.0 & 1187 & 5.1 & 4.8 & 4.8 & 4.8 \\
\hline gcut3 & 30 & 250 & 1803 & 1803 & 1803 & 1803.0 & 1803 & 0.0 & 0.0 & 0.0 & 0.0 \\
\hline gcut4 & 50 & 250 & 2934 & 3002 & 3017 & 3010.5 & 3002 & 2.3 & 2.8 & 2.6 & 2.3 \\
\hline gcut5 & 10 & 500 & 1172 & 1273 & 1273 & 1273.0 & 1273 & 8.6 & 8.6 & 8.6 & 8.6 \\
\hline gcut6 & 20 & 500 & 2514 & 2627 & 2632 & 2632.0 & 2629.5 & 4.5 & 4.7 & 4.7 & 4.6 \\
\hline gcut7 & 30 & 500 & 4641 & 4693 & 4693 & 4693.0 & 4693.8 & 1.1 & 1.1 & 1.1 & 1.1 \\
\hline gcut8 & 50 & 500 & 5703 & 5912 & 5876 & 5890.4 & 5884.2 & 3.7 & 3.0 & 3.3 & 3.2 \\
\hline gcut9 & 10 & 1000 & 2022 & 2317 & 2317 & 2317.0 & 2317 & 14.6 & 14.6 & 14.6 & 14.6 \\
\hline gcut10 & 20 & 1000 & 5356 & 5964 & 5973 & 5964.8 & 5964.9 & 11.4 & 11.5 & 11.4 & 11.4 \\
\hline gcut11 & 30 & 1000 & 6537 & 6899 & 6891 & 6884.4 & 6883.6 & 5.5 & 5.4 & 5.3 & 5.3 \\
\hline gcut12 & 50 & 1000 & 12522 & 14690 & 14690 & 14690.0 & 14690 & 17.3 & 17.3 & 17.3 & 17.3 \\
\hline gcut13 & 32 & 3000 & 4772 & 4994 & 4977 & 4965.9 & 4963.2 & 4.7 & 4.3 & 4.1 & 4.0 \\
\hline ngcut1 & 10 & 10 & 23 & 23 & 23 & 23.0 & 23 & 0.0 & 0.0 & 0.0 & 0.0 \\
\hline ngcut2 & 17 & 10 & 30 & 30 & 30 & 31.0 & 31 & 0.0 & 0.0 & 3.3 & 3.3 \\
\hline ngcut3 & 21 & 10 & 28 & 28 & 28 & 28.0 & 28 & 0.0 & 0.0 & 0.0 & 0.0 \\
\hline ngcut4 & 7 & 10 & 20 & 20 & 20 & 20.0 & 20 & 0.0 & 0.0 & 0.0 & 0.0 \\
\hline ngcut5 & 14 & 10 & 36 & 36 & 36 & 36.0 & 36 & 0.0 & 0.0 & 0.0 & 0.0 \\
\hline ngcut6 & 15 & 10 & 29 & 31 & 31 & 31.0 & 31 & 6.9 & 6.9 & 6.9 & 6.9 \\
\hline ngcut7 & 8 & 20 & 20 & 20 & 20 & 20.0 & 20 & 0.0 & 0.0 & 0.0 & 0.0 \\
\hline ngcut8 & 13 & 20 & 32 & 33 & 34 & 34.0 & 34 & 3.1 & 6.3 & 6.3 & 6.3 \\
\hline ngcut9 & 18 & 20 & 49 & 50 & 51 & 52.0 & 51 & 2.0 & 4.1 & 6.1 & 4.1 \\
\hline ngcut10 & 13 & 30 & 80 & 80 & 80 & 80.0 & 80 & 0.0 & 0.0 & 0.0 & 0.0 \\
\hline ngcut11 & 15 & 30 & 50 & 52 & 52 & 52.0 & 52 & 4.0 & 4.0 & 4.0 & 4.0 \\
\hline ngcut12 & 22 & 30 & 87 & 87 & 87 & 87.0 & 87 & 0.0 & 0.0 & 0.0 & 0.0 \\
\hline beng1 & 20 & 25 & 30 & 30 & 30 & 31.0 & 30.7 & 0.0 & 0.0 & 3.3 & 2.3 \\
\hline beng2 & 40 & 25 & 57 & 57 & 57 & 57.0 & 57 & 0.0 & 0.0 & 0.0 & 0.0 \\
\hline beng3 & 60 & 25 & 84 & 84 & 84 & 84.0 & 84 & 0.0 & 0.0 & 0.0 & 0.0 \\
\hline beng 4 & 80 & 25 & 107 & 107 & 107 & 107.0 & 107 & 0.0 & 0.0 & 0.0 & 0.0 \\
\hline beng5 & 100 & 25 & 134 & 134 & 134 & 134.0 & 134 & 0.0 & 0.0 & 0.0 & 0.0 \\
\hline beng6 & 40 & 40 & 36 & 36 & 36 & 36.0 & 36 & 0.0 & 0.0 & 0.0 & 0.0 \\
\hline beng7 & 80 & 40 & 67 & 67 & 67 & 67.0 & 67 & 0.0 & 0.0 & 0.0 & 0.0 \\
\hline beng8 & 120 & 40 & 101 & 101 & 101 & 101.0 & 101 & 0.0 & 0.0 & 0.0 & 0.0 \\
\hline beng9 & 160 & 40 & 126 & 126 & 126 & 126.0 & 126 & 0.0 & 0.0 & 0.0 & 0.0 \\
\hline beng10 & 200 & 40 & 156 & 156 & 156 & 156.0 & 156 & 0.0 & 0.0 & 0.0 & 0.0 \\
\hline \multicolumn{4}{|c|}{ Average } & 1539.95 & 1539.05 & 1538.64 & 1538.16 & 2.68 & 2.8 & 3.02 & 3.04 \\
\hline
\end{tabular}

dimensioned pieces and Pathl Path5t are of vastly differing dimensions. Nice and Path are regarded as nonzero-waste instances because the integer data are obtained by multiplying the original data by 10 and rounding to the nearest integer [16]. Nice and Path are more difficult because the optimal solutions of many instances are not known. From Table 8, GRASP, SVC, and ISA return an average gap of $1.65,0.96$, and 0.72 , respectively. HDA with an average gap of 0.63 performs better than GRASP, SVC, and ISA. HDA performs the same or better for 65 out of 72 instances, so its advantage is very obvious.

Table 9 reports results of large data set ZDF which is generated by combining zero-waste and nonzero-waste data [12]. ZDF includes several extra-large instances ZDF14 16 $(n>15000)$. Generally, it is very difficult to obtain the optimal solutions of ZDF14 16 within a reasonable time. 
TABLE 7: Results obtained by GRASP, SVC, ISA, and HDA on BWMV.

\begin{tabular}{|c|c|c|c|c|c|c|c|c|c|c|c|}
\hline \multicolumn{4}{|c|}{ Instances } & \multicolumn{4}{|c|}{ meanh } & \multicolumn{4}{|c|}{ Gap } \\
\hline & $n$ & $W$ & LB & GRASP & SVC & ISA & HDA & GRASP & SVC & ISA & HDA \\
\hline \multirow{5}{*}{$\mathrm{C} 01$} & 20 & 10 & 60.3 & 61.4 & 61.4 & 61.3 & 61.3 & 1.8 & 1.8 & 1.7 & 1.7 \\
\hline & 40 & 10 & 121.6 & 121.9 & 122 & 121.8 & 121.8 & 0.2 & 0.3 & 0.2 & 0.2 \\
\hline & 60 & 10 & 187.4 & 188.6 & 188.6 & 188.6 & 188.6 & 0.6 & 0.6 & 0.6 & 0.6 \\
\hline & 80 & 10 & 262.2 & 262.6 & 262.6 & 262.6 & 262.6 & 0.2 & 0.2 & 0.2 & 0.2 \\
\hline & 100 & 10 & 304.4 & 305 & 304.9 & 304.9 & 304.9 & 0.2 & 0.2 & 0.2 & 0.2 \\
\hline \multirow{5}{*}{$\mathrm{C} 02$} & 20 & 30 & 19.7 & 19.8 & 19.8 & 19.9 & 19.8 & 0.5 & 0.5 & 1.0 & 0.5 \\
\hline & 40 & 30 & 39.1 & 39.1 & 39.1 & 39.1 & 39.1 & 0.0 & 0.0 & 0.0 & 0.0 \\
\hline & 60 & 30 & 60.1 & 60.3 & 60.1 & 60.1 & 60.1 & 0.3 & 0.0 & 0.0 & 0.0 \\
\hline & 80 & 30 & 83.2 & 83.3 & 83.2 & 83.2 & 83.2 & 0.1 & 0.0 & 0.0 & 0.0 \\
\hline & 100 & 30 & 100.5 & 100.6 & 100.5 & 100.5 & 100.5 & 0.1 & 0.0 & 0.0 & 0.0 \\
\hline \multirow{5}{*}{$\mathrm{C} 03$} & 20 & 40 & 157.4 & 163.5 & 164.6 & 164.0 & 163.7 & 3.9 & 4.6 & 4.2 & 4.0 \\
\hline & 40 & 40 & 328.8 & 334.2 & 333.9 & 333.8 & 333.8 & 1.6 & 1.6 & 1.5 & 1.5 \\
\hline & 60 & 40 & 500 & 506.6 & 506.9 & 505.8 & 505.9 & 1.3 & 1.4 & 1.2 & 1.2 \\
\hline & 80 & 40 & 701.7 & 709.7 & 710.1 & 709.2 & 709.5 & 1.1 & 1.2 & 1.1 & 1.1 \\
\hline & 100 & 40 & 832.7 & 840.2 & 839.9 & 837.8 & 838.4 & 0.9 & 0.9 & 0.6 & 0.7 \\
\hline \multirow{5}{*}{ C04 } & 20 & 100 & 61.4 & 63.3 & 63.8 & 63.9 & 63.4 & 3.1 & 3.9 & 4.1 & 3.3 \\
\hline & 40 & 100 & 123.9 & 126.2 & 126.2 & 126.1 & 125.8 & 1.9 & 1.9 & 1.8 & 1.5 \\
\hline & 60 & 100 & 193 & 196.6 & 195.6 & 195.5 & 195.5 & 1.9 & 1.3 & 1.3 & 1.3 \\
\hline & 80 & 100 & 267.2 & 272 & 270.5 & 269.8 & 270.1 & 1.8 & 1.2 & 1.0 & 1.1 \\
\hline & 100 & 100 & 322 & 327.3 & 325.3 & 324.6 & 324.6 & 1.6 & 1.0 & 0.8 & 0.8 \\
\hline \multirow{5}{*}{ C05 } & 20 & 100 & 512.2 & 533.9 & 537.9 & 534.6 & 534.1 & 4.2 & 5.0 & 4.4 & 4.3 \\
\hline & 40 & 100 & 1053.8 & 1074.4 & 1076.4 & 1073.6 & 1073.5 & 2.0 & 2.1 & 1.9 & 1.9 \\
\hline & 60 & 100 & 1614 & 1645.5 & 1647.6 & 1643.4 & 1644.0 & 2.0 & 2.1 & 1.8 & 1.9 \\
\hline & 80 & 100 & 2268.4 & 2290.5 & 2288.9 & 2289.0 & 2289.3 & 1.0 & 0.9 & 0.9 & 0.9 \\
\hline & 100 & 100 & 2617.4 & 2651.1 & 2653.5 & 2644.4 & 2646.4 & 1.3 & 1.4 & 1.0 & 1.1 \\
\hline \multirow{5}{*}{ C06 } & 20 & 10 & 159.9 & 167.2 & 169.6 & 169.6 & 168.7 & 4.6 & 6.1 & 6.1 & 5.5 \\
\hline & 40 & 10 & 323.5 & 333.4 & 332.6 & 334.0 & 333.2 & 3.1 & 2.8 & 3.2 & 3.0 \\
\hline & 60 & 10 & 505.1 & 519.9 & 517.2 & 519.0 & 518.5 & 2.9 & 2.4 & 2.8 & 2.6 \\
\hline & 80 & 10 & 699.7 & 718.4 & 714.7 & 715.5 & 715.4 & 2.7 & 2.1 & 2.3 & 2.2 \\
\hline & 100 & 10 & 843.8 & 865.1 & 860.6 & 861.1 & 861.2 & 2.5 & 2.0 & 2.1 & 2.1 \\
\hline \multirow{5}{*}{$\mathrm{C} 07$} & 20 & 30 & 490.4 & 501.9 & 501.9 & 501.9 & 501.9 & 2.3 & 2.3 & 2.3 & 2.3 \\
\hline & 40 & 30 & 1049.7 & 1059 & 1059.9 & 1059.0 & 1059.4 & 0.9 & 1.0 & 0.9 & 0.9 \\
\hline & 60 & 30 & 1515.9 & 1529.6 & 1530 & 1529.6 & 1529.6 & 0.9 & 0.9 & 0.9 & 0.9 \\
\hline & 80 & 30 & 2206.1 & 2222.2 & 2222.1 & 2222.1 & 2222.1 & 0.7 & 0.7 & 0.7 & 0.7 \\
\hline & 100 & 30 & 2627 & 2644 & 2644 & 2645.4 & 2644.1 & 0.6 & 0.6 & 0.7 & 0.7 \\
\hline \multirow{5}{*}{ C08 } & 20 & 40 & 434.6 & 458.3 & 461.2 & 458.6 & 458.0 & 5.5 & 6.1 & 5.5 & 5.4 \\
\hline & 40 & 40 & 922 & 954.3 & 956.5 & 951.9 & 951.8 & 3.5 & 3.7 & 3.2 & 3.2 \\
\hline & 60 & 40 & 1360.9 & 1405 & 1403.5 & 1399.4 & 1403.1 & 3.2 & 3.1 & 2.8 & 3.1 \\
\hline & 80 & 40 & 1909.3 & 1971.5 & 1965 & 1954.7 & 1960.9 & 3.3 & 2.9 & 2.4 & 2.7 \\
\hline & 100 & 40 & 2362.8 & 2436.8 & 2425 & 2410.8 & 2418.3 & 3.1 & 2.6 & 2.0 & 2.4 \\
\hline \multirow{5}{*}{ C09 } & 20 & 100 & 1106.8 & 1106.8 & 1106.8 & 1106.8 & 1106.8 & 0.0 & 0.0 & 0.0 & 0.0 \\
\hline & 40 & 100 & 2189.2 & 2190.6 & 2190.6 & 2190.6 & 2191.1 & 0.1 & 0.1 & 0.1 & 0.1 \\
\hline & 60 & 100 & 3410.4 & 3410.4 & 3410.4 & 3410.4 & 3410.4 & 0.0 & 0.0 & 0.0 & 0.0 \\
\hline & 80 & 100 & 4578.6 & 4588.1 & 4588.1 & 4588.1 & 4588.1 & 0.2 & 0.2 & 0.2 & 0.2 \\
\hline & 100 & 100 & 5430.5 & 5434.9 & 5434.9 & 5434.9 & 5434.9 & 0.1 & 0.1 & 0.1 & 0.1 \\
\hline \multirow{5}{*}{$\mathrm{C} 10$} & 20 & 100 & 337.8 & 350.5 & 351.5 & 350.4 & 350.1 & 3.8 & 4.1 & 3.7 & 3.6 \\
\hline & 40 & 100 & 642.8 & 664.4 & 667 & 664.0 & 663.7 & 3.4 & 3.8 & 3.3 & 3.3 \\
\hline & 60 & 100 & 911.1 & 934.7 & 936.6 & 933.1 & 933.2 & 2.6 & 2.8 & 2.4 & 2.4 \\
\hline & 80 & 100 & 1177.6 & 1209.9 & 1212.4 & 1204.1 & 1205.2 & 2.7 & 3.0 & 2.3 & 2.3 \\
\hline & 100 & 100 & 1476.5 & 1512.3 & 1514 & 1504.2 & 1506.5 & 2.4 & 2.5 & 1.9 & 2.0 \\
\hline \multicolumn{4}{|c|}{ Average } & 1043.34 & 1043.19 & 1041.53 & 1041.92 & 1.77 & 1.80 & 1.66 & 1.63 \\
\hline
\end{tabular}


TABLE 8: Results obtained by GRASP, SVC, ISA, and HDA on Nice and Path.

\begin{tabular}{|c|c|c|c|c|c|c|c|c|c|c|c|}
\hline \multicolumn{4}{|c|}{ Instance } & \multicolumn{4}{|c|}{ meanh } & \multicolumn{4}{|c|}{ Gap } \\
\hline & $n$ & $W$ & LB & GRASP & SVC & ISA & HDA & GRASP & SVC & ISA & HDA \\
\hline Nicel & 25 & 1000 & 1000 & 1034 & 1037 & 1040.7 & 1034.8 & 3.4 & 3.7 & 4.1 & 3.5 \\
\hline Nice2 & 50 & 1000 & 1001 & 1047 & 1038 & 1047.2 & 1037.7 & 4.6 & 3.7 & 4.6 & 3.7 \\
\hline Nice3 & 100 & 1000 & 1001 & 1041 & 1035 & 1036.5 & 1030.9 & 4.0 & 3.4 & 3.5 & 3.0 \\
\hline Nice4 & 200 & 1000 & 1001 & 1037 & 1026 & 1030.9 & 1023 & 3.6 & 2.5 & 2.9 & 2.2 \\
\hline Nice5 & 500 & 1000 & 1000 & 1024 & 1017 & 1015.0 & 1008 & 2.4 & 1.7 & 1.5 & 0.8 \\
\hline Nice6 & 1000 & 1000 & 999 & 1020 & 1014 & 1011.0 & 1004 & 2.1 & 1.5 & 1.2 & 0.5 \\
\hline \multirow{10}{*}{ Nicelt } & 1000 & 1000 & 1001 & 1026 & 1015 & 1011.0 & 1006 & 2.5 & 1.4 & 1.0 & 0.5 \\
\hline & 1000 & 1000 & 1001 & 1022 & 1016 & 1010.0 & 1005 & 2.1 & 1.5 & 0.9 & 0.4 \\
\hline & 1000 & 1000 & 1000 & 1020 & 1013 & 1011.0 & 1005 & 2.0 & 1.3 & 1.1 & 0.5 \\
\hline & 1000 & 1000 & 1000 & 1019 & 1013 & 1010.0 & 1005 & 1.9 & 1.3 & 1.0 & 0.5 \\
\hline & 1000 & 1000 & 1000 & 1022 & 1014 & 1010.0 & 1005 & 2.2 & 1.4 & 1.0 & 0.5 \\
\hline & 1000 & 1000 & 1001 & 1020 & 1014 & 1010.0 & 1005 & 1.9 & 1.3 & 0.9 & 0.4 \\
\hline & 1000 & 1000 & 1000 & 1022 & 1014 & 1010.0 & 1006 & 2.2 & 1.4 & 1.0 & 0.6 \\
\hline & 1000 & 1000 & 1001 & 1021 & 1016 & 1012.0 & 1007 & 2.0 & 1.5 & 1.1 & 0.6 \\
\hline & 1000 & 1000 & 1000 & 1022 & 1017 & 1012.0 & 1005 & 2.2 & 1.7 & 1.2 & 0.5 \\
\hline & 1000 & 1000 & 1001 & 1027 & 1016 & 1012.0 & 1007 & 2.6 & 1.5 & 1.1 & 0.6 \\
\hline \multirow{10}{*}{ Nice2t } & 2000 & 1000 & 1001 & 1016 & 1008 & 1006.0 & 1004 & 1.5 & 0.7 & 0.5 & 0.3 \\
\hline & 2000 & 1000 & 1001 & 1015 & 1011 & 1005.0 & 1005 & 1.4 & 1.0 & 0.4 & 0.4 \\
\hline & 2000 & 1000 & 1000 & 1016 & 1008 & 1007.0 & 1005 & 1.6 & 0.8 & 0.7 & 0.5 \\
\hline & 2000 & 1000 & 1000 & 1014 & 1007 & 1006.0 & 1003 & 1.4 & 0.7 & 0.6 & 0.3 \\
\hline & 2000 & 1000 & 1000 & 1015 & 1008 & 1006.0 & 1003 & 1.5 & 0.8 & 0.6 & 0.3 \\
\hline & 2000 & 1000 & 1000 & 1016 & 1002 & 1005.0 & 1004 & 1.6 & 0.2 & 0.5 & 0.4 \\
\hline & 2000 & 1000 & 1001 & 1016 & 1007 & 1007.0 & 1004 & 1.5 & 0.6 & 0.6 & 0.3 \\
\hline & 2000 & 1000 & 1001 & 1014 & 1006 & 1006.0 & 1003 & 1.3 & 0.5 & 0.5 & 0.2 \\
\hline & 2000 & 1000 & 1001 & 1016 & 1008 & 1007.0 & 1006 & 1.5 & 0.7 & 0.6 & 0.5 \\
\hline & 2000 & 1000 & 1001 & 1016 & 1009 & 1007.0 & 1005 & 1.5 & 0.8 & 0.6 & 0.4 \\
\hline \multirow{10}{*}{ Nice5t } & 5000 & 1000 & 1000 & 1010 & 1003 & 1003.0 & 1003 & 1.0 & 0.3 & 0.3 & 0.3 \\
\hline & 5000 & 1000 & 1001 & 1011 & 1005 & 1003.0 & 1003 & 1.0 & 0.4 & 0.2 & 0.2 \\
\hline & 5000 & 1000 & 1001 & 1010 & 1002 & 1003.0 & 1003 & 0.9 & 0.1 & 0.2 & 0.2 \\
\hline & 5000 & 1000 & 1000 & 1009 & 1005 & 1002.0 & 1003 & 0.9 & 0.5 & 0.2 & 0.3 \\
\hline & 5000 & 1000 & 1001 & 1011 & 1006 & 1003.0 & 1004 & 1.0 & 0.5 & 0.2 & 0.3 \\
\hline & 5000 & 1000 & 1000 & 1009 & 1001 & 1002.0 & 1001 & 0.9 & 0.1 & 0.2 & 0.1 \\
\hline & 5000 & 1000 & 1001 & 1011 & 1004 & 1003.0 & 1003 & 1.0 & 0.3 & 0.2 & 0.2 \\
\hline & 5000 & 1000 & 1000 & 1011 & 1004 & 1002.0 & 1003 & 1.1 & 0.4 & 0.2 & 0.3 \\
\hline & 5000 & 1000 & 1001 & 1010 & 1004 & 1003.0 & 1003 & 0.9 & 0.3 & 0.2 & 0.2 \\
\hline & 5000 & 1000 & 1000 & 1010 & 1005 & 1003.0 & 1003 & 1.0 & 0.5 & 0.3 & 0.3 \\
\hline Path1 & 25 & 1000 & 1001 & 1042 & 1042 & 1042.0 & 1041.8 & 4.1 & 4.1 & 4.1 & 4.1 \\
\hline Path2 & 50 & 1000 & 1000 & 1019 & 1014 & 1014.7 & 1011.9 & 1.9 & 1.4 & 1.5 & 1.2 \\
\hline Path3 & 100 & 1000 & 1000 & 1027 & 1022 & 1022.6 & 1025.1 & 2.7 & 2.2 & 2.3 & 2.5 \\
\hline Path4 & 200 & 1000 & 1002 & 1023 & 1018 & 1017.7 & 1013 & 2.1 & 1.6 & 1.6 & 1.1 \\
\hline Path5 & 500 & 1000 & 1000 & 1034 & 1022 & 1020.0 & 1016 & 3.4 & 2.2 & 2.0 & 1.6 \\
\hline Path6 & 1000 & 1000 & 1002 & 1026 & 1018 & 1011.0 & 1010 & 2.4 & 1.6 & 0.9 & 0.8 \\
\hline \multirow{10}{*}{ Pathlt } & 1000 & 1000 & 999 & 1019 & 1011 & 1007.0 & 1003 & 2.0 & 1.2 & 0.8 & 0.4 \\
\hline & 1000 & 1000 & 1001 & 1018 & 1010 & 1006.0 & 1005 & 1.7 & 0.9 & 0.5 & 0.4 \\
\hline & 1000 & 1000 & 1001 & 1018 & 1013 & 1008.0 & 1006 & 1.7 & 1.2 & 0.7 & 0.5 \\
\hline & 1000 & 1000 & 1000 & 1016 & 1009 & 1006.0 & 1003 & 1.6 & 0.9 & 0.6 & 0.3 \\
\hline & 1000 & 1000 & 1003 & 1024 & 1017 & 1010.0 & 1010 & 2.1 & 1.4 & 0.7 & 0.7 \\
\hline & 1000 & 1000 & 1002 & 1018 & 1013 & 1010.0 & 1005 & 1.6 & 1.1 & 0.8 & 0.3 \\
\hline & 1000 & 1000 & 999 & 1019 & 1012 & 1008.0 & 1004 & 2.0 & 1.3 & 0.9 & 0.5 \\
\hline & 1000 & 1000 & 1000 & 1020 & 1012 & 1008.0 & 1006 & 2.0 & 1.2 & 0.8 & 0.6 \\
\hline & 1000 & 1000 & 999 & 1019 & 1012 & 1006.0 & 1003 & 2.0 & 1.3 & 0.7 & 0.4 \\
\hline & 1000 & 1000 & 1002 & 1018 & 1011 & 1008.0 & 1006 & 1.6 & 0.9 & 0.6 & 0.4 \\
\hline
\end{tabular}


TABLE 8: Continued.

\begin{tabular}{|c|c|c|c|c|c|c|c|c|c|c|c|}
\hline \multicolumn{4}{|c|}{ Instance } & \multicolumn{4}{|c|}{ meanh } & \multicolumn{4}{|c|}{ Gap } \\
\hline & $n$ & $W$ & LB & GRASP & SVC & ISA & HDA & GRASP & SVC & ISA & HDA \\
\hline \multirow{10}{*}{ Path2t } & 2000 & 1000 & 1000 & 1015 & 1009 & 1006.0 & 1002 & 1.5 & 0.9 & 0.6 & 0.2 \\
\hline & 2000 & 1000 & 1002 & 1016 & 1010 & 1007.0 & 1004 & 1.4 & 0.8 & 0.5 & 0.2 \\
\hline & 2000 & 1000 & 1000 & 1015 & 1011 & 1006.0 & 1003 & 1.5 & 1.1 & 0.6 & 0.3 \\
\hline & 2000 & 1000 & 999 & 1014 & 1007 & 1003.0 & 1000 & 1.5 & 0.8 & 0.4 & 0.1 \\
\hline & 2000 & 1000 & 1002 & 1018 & 1012 & 1008.0 & 1006 & 1.6 & 1.0 & 0.6 & 0.4 \\
\hline & 2000 & 1000 & 1002 & 1016 & 1011 & 1007.0 & 1003 & 1.4 & 0.9 & 0.5 & 0.1 \\
\hline & 2000 & 1000 & 998 & 1011 & 1007 & 1004.0 & 1001 & 1.3 & 0.9 & 0.6 & 0.3 \\
\hline & 2000 & 1000 & 998 & 1014 & 1010 & 1004.0 & 1003 & 1.6 & 1.2 & 0.6 & 0.5 \\
\hline & 2000 & 1000 & 1001 & 1017 & 1010 & 1008.0 & 1003 & 1.6 & 0.9 & 0.7 & 0.2 \\
\hline & 2000 & 1000 & 1003 & 1018 & 1009 & 1009.0 & 1005 & 1.5 & 0.6 & 0.6 & 0.2 \\
\hline \multirow{10}{*}{ Path5t } & 5000 & 1000 & 1000 & 1010 & 1002 & 1003.0 & 1001 & 1.0 & 0.2 & 0.3 & 0.1 \\
\hline & 5000 & 1000 & 998 & 1009 & 1003 & 1001.0 & 1001 & 1.1 & 0.5 & 0.3 & 0.3 \\
\hline & 5000 & 1000 & 1000 & 1011 & 1002 & 1003.0 & 1002 & 1.1 & 0.2 & 0.3 & 0.2 \\
\hline & 5000 & 1000 & 995 & 1006 & 998 & 997.0 & 997 & 1.1 & 0.3 & 0.2 & 0.2 \\
\hline & 5000 & 1000 & 1004 & 1016 & 1005 & 1006.0 & 1006 & 1.2 & 0.1 & 0.2 & 0.2 \\
\hline & 5000 & 1000 & 1000 & 1009 & 1003 & 1002.0 & 1001 & 0.9 & 0.3 & 0.2 & 0.1 \\
\hline & 5000 & 1000 & 998 & 1009 & 1001 & 1001.0 & 1000 & 1.1 & 0.3 & 0.3 & 0.2 \\
\hline & 5000 & 1000 & 996 & 1007 & 998 & 999.0 & 998 & 1.1 & 0.2 & 0.3 & 0.2 \\
\hline & 5000 & 1000 & 997 & 1007 & 999 & 999.0 & 998 & 1.0 & 0.2 & 0.2 & 0.1 \\
\hline & 5000 & 1000 & 1002 & 1013 & 1004 & 1004.0 & 1005 & 1.1 & 0.2 & 0.2 & 0.3 \\
\hline \multicolumn{4}{|c|}{ Average } & 1016.63 & 1009.75 & 1007.36 & 1006.57 & 1.65 & 0.96 & 0.72 & 0.63 \\
\hline
\end{tabular}

TABLE 9: Results obtained by GRASP, SVC, ISA, and HDA on ZDF.

\begin{tabular}{|c|c|c|c|c|c|c|c|c|c|c|c|}
\hline \multicolumn{4}{|c|}{ Instance } & \multicolumn{4}{|c|}{ meanh } & \multicolumn{4}{|c|}{ Gap } \\
\hline & $n$ & $W$ & LB & GRASP & SVC & ISA & HDA & GRASP & SVC & ISA & HDA \\
\hline zdf1 & 580 & 100 & 330 & 333 & 331 & 330.0 & 330 & 0.9 & 0.3 & 0.0 & 0 \\
\hline $\mathrm{zdf} 2$ & 660 & 100 & 357 & 360 & 358 & 357.0 & 357 & 0.8 & 0.3 & 0.0 & 0 \\
\hline zdf3 & 740 & 100 & 384 & 387 & 385 & 384.0 & 384 & 0.8 & 0.3 & 0.0 & 0 \\
\hline $\mathrm{zdf} 4$ & 820 & 100 & 407 & 410 & 408 & 407.0 & 407 & 0.7 & 0.2 & 0.0 & 0 \\
\hline zdf5 & 900 & 100 & 434 & 437 & 434 & 434.0 & 434 & 0.7 & 0.0 & 0.0 & 0 \\
\hline zdf6 & 1532 & 3000 & 4872 & 5251 & 5085 & 5081.8 & 5066 & 7.8 & 4.4 & 4.3 & 4 \\
\hline zdf7 & 2432 & 3000 & 4852 & 5163 & 5083 & 5084.7 & 5017 & 6.4 & 4.8 & 4.8 & 3.4 \\
\hline zdf8 & 2532 & 3000 & 5172 & 5544 & 5386 & 5549.0 & 5397 & 7.2 & 4.1 & 7.3 & 4.4 \\
\hline zdf9 & 5032 & 3000 & 5172 & 5476 & 5468 & 5404.0 & 5408 & 5.9 & 5.7 & 4.5 & 4.6 \\
\hline zdf10 & 5064 & 6000 & 5172 & 5570 & 5462 & 5419.0 & 5433 & 7.7 & 5.6 & 4.8 & 5 \\
\hline zdf11 & 7564 & 6000 & 5172 & 5562 & 5516 & 5419.0 & 5439 & 7.5 & 6.7 & 4.8 & 5.2 \\
\hline zdf12 & 10064 & 6000 & 5172 & - & 5651 & 5454.0 & 5403 & - & 9.3 & 5.5 & 4.5 \\
\hline zdf13 & 15096 & 9000 & 5172 & - & 5600 & 5415.0 & 5415 & - & 8.3 & 4.7 & 4.7 \\
\hline zdf14 & 25032 & 3000 & 5172 & - & 5468 & 5286.0 & 5353 & - & 5.7 & 2.2 & 3.5 \\
\hline zdf15 & 50032 & 3000 & 5172 & - & 5960 & 5172.0 & 5273 & - & 15.2 & 0.0 & 2 \\
\hline zdf16 & 75032 & 3000 & 5172 & - & 5931 & 5172.0 & 5203 & - & 14.7 & 0.0 & 0.6 \\
\hline \multicolumn{4}{|c|}{ Average } & & 3907.88 & 3773.03 & 3769.94 & 5.34 & & 2.67 & 2.62 \\
\hline
\end{tabular}

The symbol “-” denotes that the GRASP executable program cannot return the results, so its average gap is not given. From Table 9, we can observe that SVC and ISA return an average gap of 5.34 and 2.67, respectively. HDA with an average gap of 2.62 performs better than SVC and ISA. ISA and HDA perform well for large instances.

\section{Conclusions}

A hybrid demon algorithm for 2SP is presented in this paper. This algorithm improves the scoring rule presented by Leung et al. [18] and a demon algorithm with one parameter is used to improve the quality of the solutions. Computational results 


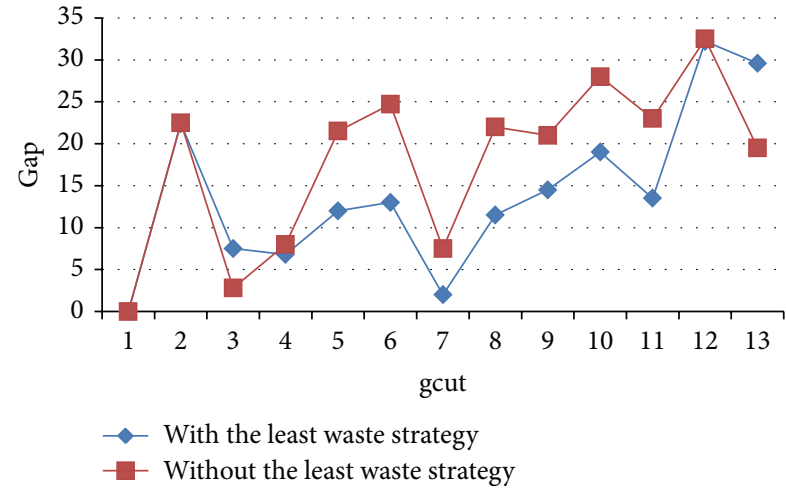

FIGURE 8: Effect of the least waste strategy on the data set gcut.

have shown that HDA outperforms well-known GRASP, SVC, and ISA. The experiments in Section 3 show that HDA is more stable and efficient for different $D$ value. HDA performs well for large instances; future work is to further improve the quality of the solutions by combining exact algorithms or other metaheuristic algorithms.

\section{Conflict of Interests}

The authors declare that there is no conflict of interests regarding the publication of this paper.

\section{Acknowledgments}

This work was supported by the National Nature Science Foundation of China (Grant no. 71272085).

\section{References}

[1] A. Lodi, S. Martello, and M. Monaci, "Two-dimensional packing problems: a survey," European Journal of Operational Research, vol. 141, no. 2, pp. 241-252, 2002.

[2] E. Hopper and B. C. H. Turton, "A review of the application of meta-heuristic algorithms to 2D strip packing problems," Artificial Intelligence Review, vol. 16, no. 4, pp. 257-300, 2001.

[3] G. Wäscher, H. Haußner, and H. Schumann, "An improved typology of cutting and packing problems," European Journal of Operational Research, vol. 183, no. 3, pp. 1109-1130, 2007.

[4] S. Martello, M. Monaci, and D. Vigo, "An exact approach to the strip-packing problem," INFORMS Journal on Computing, vol. 15, no. 3, pp. 310-319, 2003.

[5] M. Kenmochi, T. Imamichi, K. Nonobe, M. Yagiura, and H. Nagamochi, "Exact algorithms for the two-dimensional strip packing problem with and without rotations," European Journal of Operational Research, vol. 198, no. 1, pp. 73-83, 2009.

[6] B. S. Baker, J. G. Coffman Jr., and R. L. Rivest, "Orthogonal packings in two dimensions," SIAM Journal on Computing, vol. 9, no. 4, pp. 846-855, 1980.

[7] S. Jakobs, "On genetic algorithms for the packing of polygons," European Journal of Operational Research, vol. 88, no. 1, pp. 165181, 1996.
[8] E. K. Burke, G. Kendall, and G. Whitwell, "A new placement heuristic for the orthogonal stock-cutting problem," Operations Research, vol. 52, no. 4, pp. 655-672, 2004.

[9] D. Zhang, Y. Kang, and A. Deng, "A new heuristic recursive algorithm for the strip rectangular packing problem," Computers and Operations Research, vol. 33, no. 8, pp. 2209-2217, 2006.

[10] D. Zhang, S. Han, and W. Ye, "A bricklaying heuristic algorithm for the orthogonal rectangular packing problem," Chinese Journal of Computers, vol. 23, no. 3, pp. 509-515, 2008.

[11] L. Wei, D. Zhang, and Q. Chen, "A least wasted first heuristic algorithm for the rectangular packing problem," Computers and Operations Research, vol. 36, no. 5, pp. 1608-1614, 2009.

[12] S. C. H. Leung and D. Zhang, "A fast layer-based heuristic for non-guillotine strip packing," Expert Systems with Applications, vol. 38, no. 10, pp. 13032-13042, 2011.

[13] A. Bortfeldt, "A genetic algorithm for the two-dimensional strip packing problem with rectangular pieces," European Journal of Operational Research, vol. 172, no. 3, pp. 814-837, 2006.

[14] E. Hopper and B. C. H. Turton, "An empirical investigation of meta-heuristic and heuristic algorithms for a $2 \mathrm{D}$ packing problem," European Journal of Operational Research, vol. 128, no. 1, pp. 34-57, 2001.

[15] N. Lesh, J. Marks, A. McMahon, and M. Mitzenmacher, "New heuristic and interactive approaches to $2 \mathrm{D}$ rectangular strip packing," Journal of Experimental Algorithmics, vol. 10, article $1.2,2005$

[16] R. Alvarez-Valdes, F. Parreño, and J. M. Tamarit, "Reactive GRASP for the strip-packing problem," Computers and Operations Research, vol. 35, no. 4, pp. 1065-1083, 2008.

[17] E. K. Burke, G. Kendall, and G. Whitwell, "A simulated annealing enhancement of the best-fit heuristic for the orthogonal stock-cutting problem," INFORMS Journal on Computing, vol. 21, no. 3, pp. 505-516, 2009.

[18] S. C. H. Leung, D. Zhang, and K. M. Sim, "A two-stage intelligent search algorithm for the two-dimensional strip packing problem," European Journal of Operational Research, vol. 215, no. 1, pp. 57-69, 2011.

[19] G. Belov, G. Scheithauer, and E. A. Mukhacheva, "Onedimensional heuristics adapted for two-dimensional rectangular strip packing," Journal of the Operational Research Society, vol. 59, no. 6, pp. 823-832, 2008.

[20] E. K. Burke, M. R. Hyde, and G. Kendall, "A squeaky wheel optimisation methodology for two-dimensional strip packing," Computers \& Operations Research, vol. 38, no. 7, pp. 1035-1044, 2011.

[21] D. Zhang, L. Wei, S. C. H. Leung, and Q. Chen, "A binary search heuristic algorithm based on randomized local search for the rectangular strip-packing problem," INFORMS Journal on Computing, vol. 25, no. 2, pp. 332-345, 2013.

[22] F. G. Ortmann and J. H. van Vuuren, "Modified strip packing heuristics for the rectangular variable-sized bin packing problem," ORiON, vol. 26, no. 1, pp. 21-44, 2010.

[23] S. Hong, D. Zhang, H. C. Lau, X. Zeng, and Y.-W. Si, "A hybrid heuristic algorithm for the $2 \mathrm{D}$ variable-sized bin packing problem," European Journal of Operational Research, vol. 238, no. 1, pp. 95-103, 2014.

[24] E. Pinto and J. F. Oliveira, "Algorithm based on graphs for the non-guillotinable two-dimensional packing problem," in Proceedings of the 2nd ESICUP Meeting, Southampton, UK, April 2005. 
[25] J. E. Beasley, "An exact two-dimensional nonguillotine cutting tree search procedure," Operations Research, vol. 33, no. 1, pp. 49-64, 1985.

[26] J. E. Beasley, "Algorithms for unconstrained two-dimensional guillotine cutting," Journal of the Operational Research Society, vol. 36, no. 4, pp. 297-306, 1985.

[27] N. Christofides and C. Whitlock, "An algorithm for two-dimensional cutting problems," Operations Research, vol. 25, pp. 3044, 1977.

[28] B. E. Bengtsson, "Packing rectangular pieces-a heuristic approach," The Computer Journal, vol. 25, no. 3, pp. 353-357, 1982.

[29] J. O. Berkey and P. Y. Wang, "Two-dimensional finite bin packing algorithms," Journal of the Operational Research Society, vol. 38, no. 5, pp. 423-429, 1987.

[30] S. Martello and D. Vigo, "Exact solution of the two-dimensional finite bin packing problem," Management Science, vol. 44, no. 3, pp. 388-399, 1998.

[31] C. L. Valenzuela and P. Y. Wang, "Heuristics for large strip packing problems with guillotine patterns: an empirical study," in Proceedings of the 4th Metaheuristics International Conference, pp. 417-421, University of Porto, Porto, Portugal, 2001. 


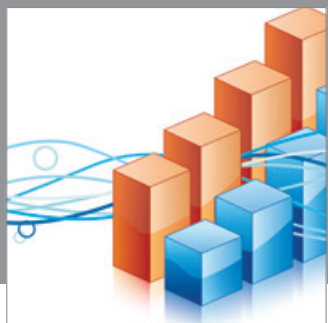

Advances in

Operations Research

mansans

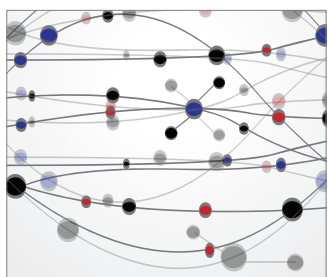

The Scientific World Journal
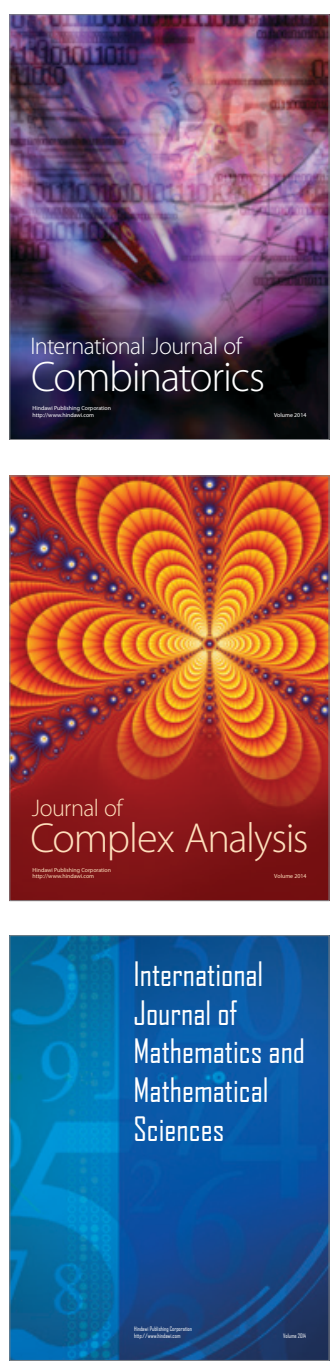
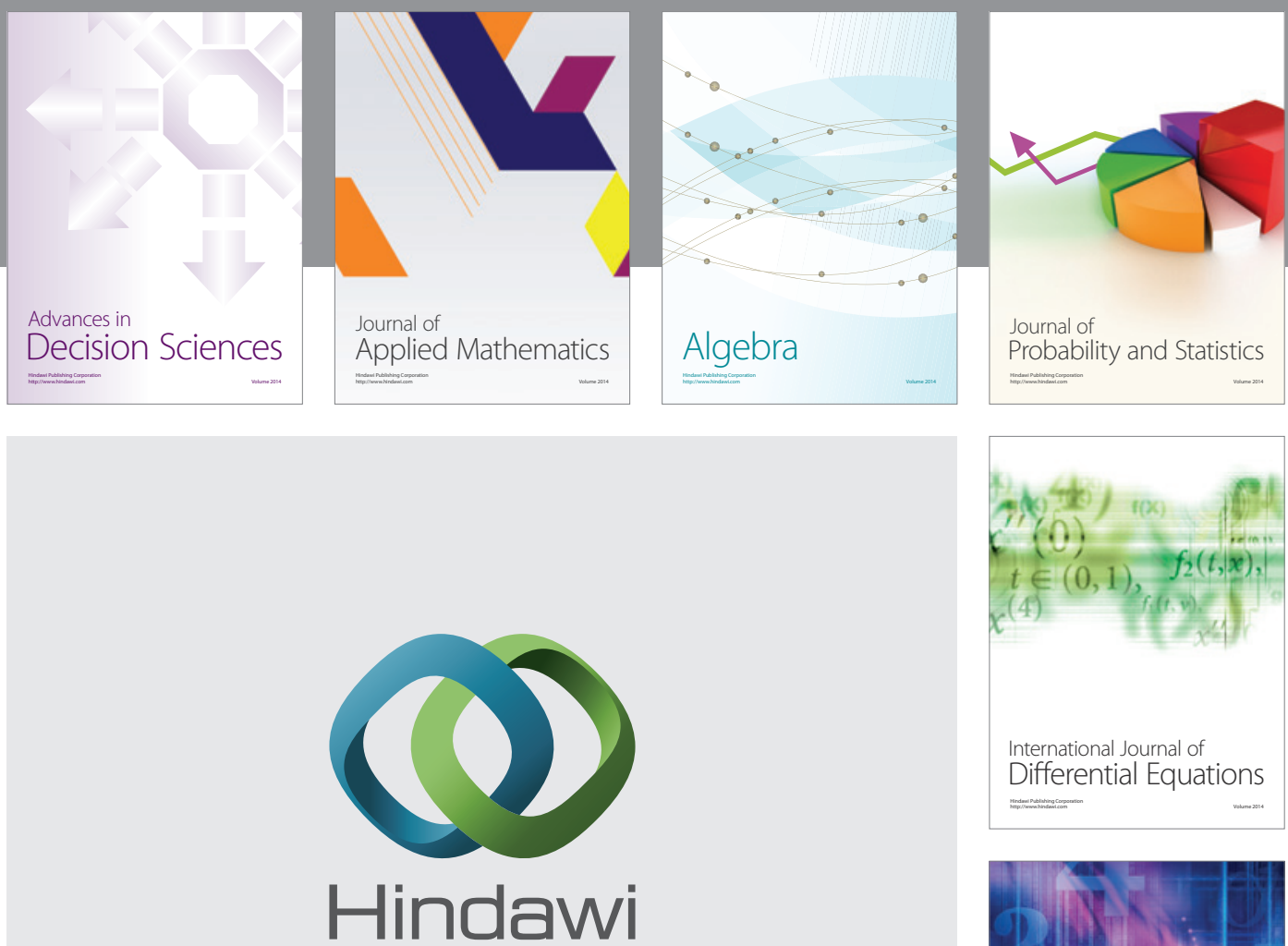

Submit your manuscripts at http://www.hindawi.com
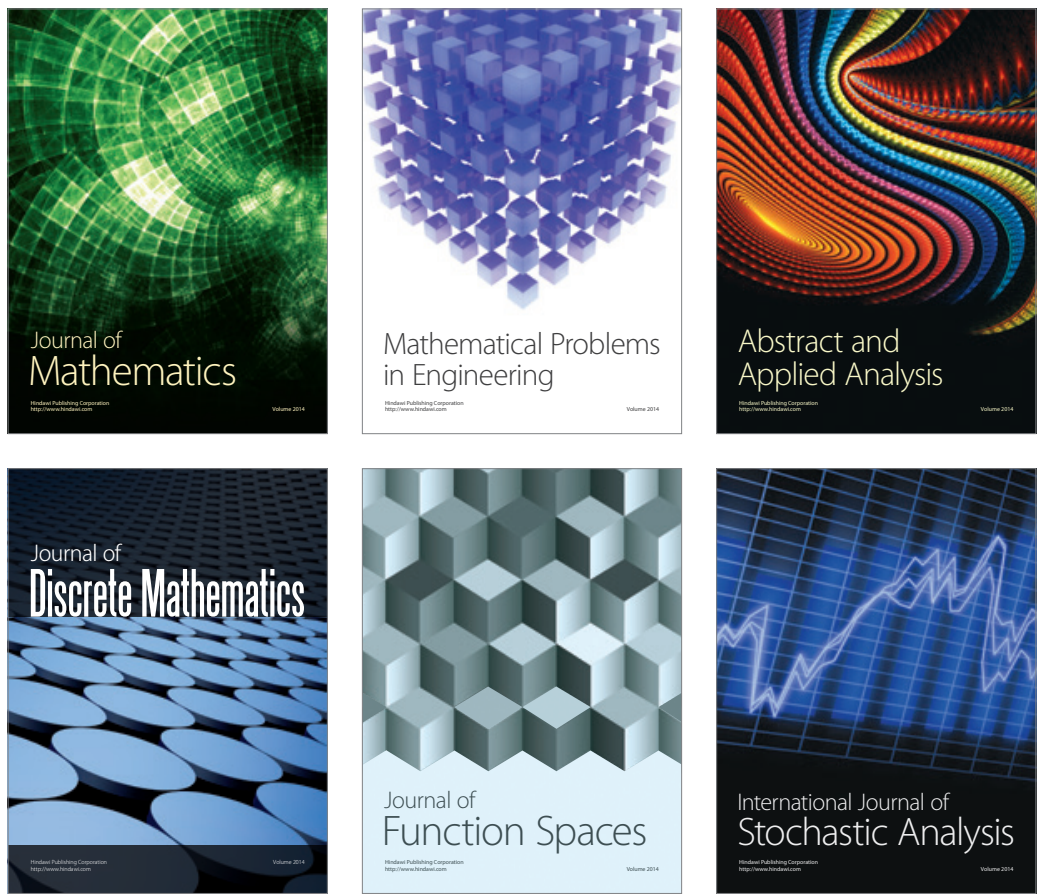

Journal of

Function Spaces

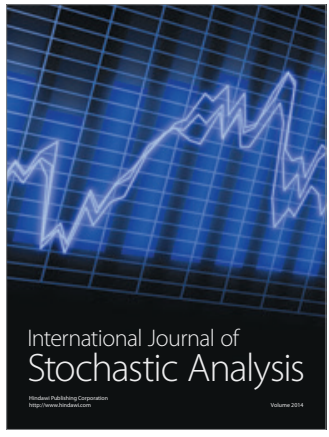

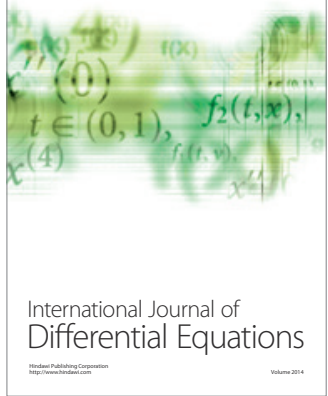
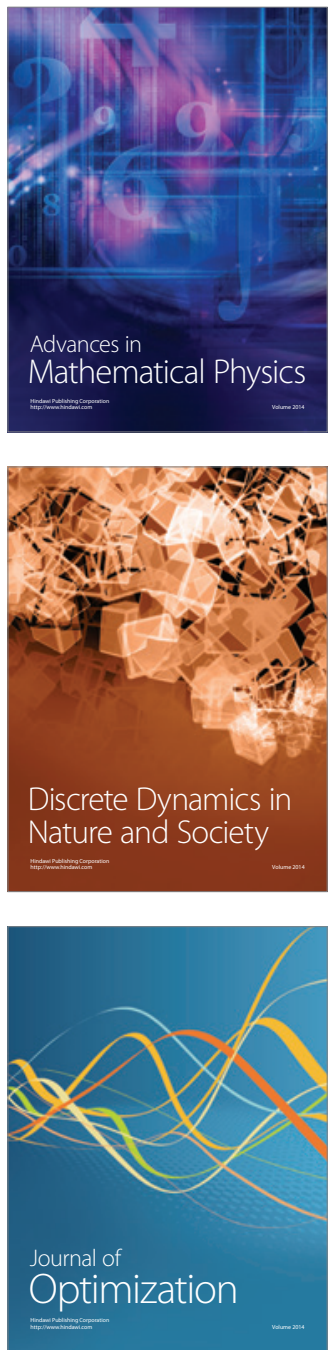